\title{
Unbalance Compensation of a Full Scale Test Rig Designed for HTR-10GT: A Frequency-Domain Approach Based on Iterative Learning Control
}

\author{
Ying He, Lei Shi, Zhengang Shi, and Zhe Sun \\ Institute of Nuclear and New Energy Technology of Tsinghua University, Collaborative Innovation Center of \\ Advanced Nuclear Energy Technology, Key Laboratory of Advanced Reactor Engineering and Safety of Ministry of Education, \\ Beijing 100084, China \\ Correspondence should be addressed to Zhe Sun; sun_zhe@tsinghua.edu.cn
}

Received 6 September 2016; Accepted 7 December 2016; Published 26 January 2017

Academic Editor: Arkady Serikov

Copyright (c) 2017 Ying He et al. This is an open access article distributed under the Creative Commons Attribution License, which permits unrestricted use, distribution, and reproduction in any medium, provided the original work is properly cited.

Unbalance vibrations are crucial problems in heavy rotational machinery, especially for the systems with high operation speed, like turbine machinery. For the program of $10 \mathrm{MW}$ High Temperature gas-cooled Reactor with direct Gas-Turbine cycle (HTR-10GT), the rated operation speed of the turbine system is 15000 RPM which is beyond the second bending frequency. In that case, even a small residual mass will lead to large unbalance vibrations. Thus, it is of great significance to study balancing methods for the system. As the turbine rotor is designed to be suspended by active magnetic bearings (AMBs), unbalance compensation could be achieved by adequate control strategies. In the paper, unbalance compensation for the Multi-Input and Multi-Output (MIMO) active magnetic bearing (AMB) system using frequency-domain iterative learning control (ILC) is analyzed. Based on the analysis, an ILC controller for unbalance compensation of the full scale test rig, which is designed for the rotor and AMBs in HTR-10GT, is designed. Simulation results are reported which show the efficiency of the ILC controller for attenuating the unbalance vibration of the full scale test rig. This research can offer valuable design criterion for unbalance compensation of the turbine machinery in HTR-10GT.

\section{Introduction}

It has been proved that the High Temperature gas-cooled Reactor (HTR) with direct energy conversion cycle (the closed Brayton cycle) has higher electricity generation efficiency [1]. With the success of $10 \mathrm{MW}$ High Temperature gas-cooled Reactor (HTR-10), the program of $10 \mathrm{MW}$ High Temperature gas-cooled Reactor with direct Gas-Turbine cycle (HTR-10GT) is proposed [2]. To meet the specific requirements for the bearings, active magnetic bearings (AMBs) are adopted to support the rotors in the Power Conversion Unit (PCU) of HTR-10GT. As illustrated in Figure 1, the PCU consists of three parts: the exciter, the generator, and the turbine. All rotors are fitted vertically and the turbine component is settled at the bottom. Aiming at studying and developing the key technologies of the turbine system, a full scale test rig for the turbine rotor and AMBs is established in compliance with the dynamic similarity principle by Institute of Nuclear and New Energy Technology (INET), Tsinghua University [3].

Rotor dynamic analysis shows that the rated operation speed of the turbine compressor rotor is beyond the second bending frequency. In that case, unbalance vibrations will be a crucial problem which will reduce the system performances and even affect the safety of the system under some circumstances. Therefore, it is absolutely essential to study the attenuation of unbalance vibrations. The traditional methods for balancing of the rotating masses are based on the principle which shifts the mass center to the geometrical center by adding or moving additional masses to or from the rotating parts. During the balancing process, repeated startup and shutdown are required, which is time-consuming and even causes failure under conditions where the rotor systems are not allowed to stop. Another disadvantage is that the 


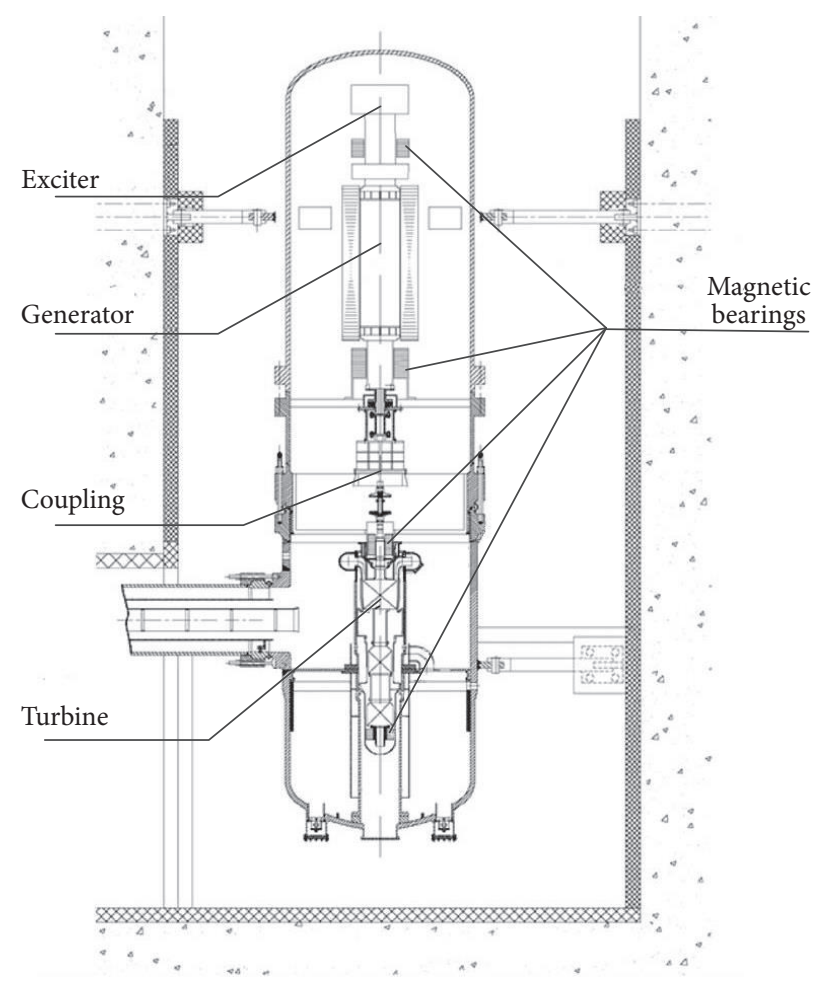

FIgURE 1: The layout of PCU in HTR-10GT.

application of the methods is limited by a balancing speed. The balanced rotor will be unbalanced if the operation speed exceeds the balancing speed; then rebalancing is required.

Another principle for balancing of the rotating masses is offsetting the unbalance forces by adding additional forces, which are of same magnitudes but opposite phases as the resultant centrifugal forces. As AMBs have specific active control ability, the additional forces for counteracting of the resultant centrifugal forces can be generated by adequate control strategies. At present, varies control methods have been proposed for unbalance compensation as reported in literatures [4-7]. However, most of the control methods are complex in strategies or required online calculation or highly rely on accurate model of the system.

In 1978, iterative learning control (ILC) was first raised by Uchiyama to achieve better control performance for high speed movement of manipulators [8]. The method possess some highly desirable features, structural simplicity, offline calculation, and almost model-independent design, since the control signal is built iteratively by using the error signal and control signal in the previous iteration. Currently, large amounts of papers related to ILC have been published. The publications are related to various issues, such as convergency analysis $[9,10]$, survey paper [11-13], and practical application [14-16]. For practical application, many fields are included. Comprehensive reviews of applications in energy generation, healthcare, and lasers could be found in [17]. In [15], two model-based ILC algorithms (Arm Side Measurement ILC and Force Measurement ILC) were successfully applied to an industrial robot for increasing the accuracy of robotic machining. In [14], ILC control was used to achieve accurate and high speed bidirectional scanning for high speed laser scanning microscopy and the ILC controller was designed by two inversion methods: the zero phase approximation and time delay approximation. Another successfully application is for reducing runout in hard disk drives. In [16], ILC was used to design a reference signal to reduce periodic disturbances and the method was tested on a hard disk drive.

The goal for this paper is to develop ILC controller for unbalance compensation of an AMB system. A small amount of papers related to this topic has been reported. The ILC controller can be designed either by time-domain approach or by frequency-domain approach. In [18], frequency-domain ILC controller was designed and implemented successfully for unbalance compensation of a rotating system with a single active magnetic bearing (AMB) controlled from one end. However, in the paper, the system was simplified as totally decoupled system and the control strategy was stated from the viewpoint of single-input and single-output (SISO) system. Bi et al. proposed a control scheme named after automatic learning control (ALC), based on time-domain iterative learning control and scheduled gain control, to compensate the unbalance effect in AMB system $[19,20]$. The same as literature [18], in the paper, the ALC was designed for four one-DOF subsystems. That is to say, the AMB system was decoupled as SISO systems.

In the paper, we focus on analyzing unbalance compensation for Multi-Input Multi-Output (MIMO) AMB system using the frequency-domain approach based ILC controller. Based on the analysis, an ILC controller is designed for unbalance compensation of the full scale test rig which is designed for HTR-10GT. The simulation results show that the unbalance vibrations of the rotor are reduced significantly, which validates the efficiency of the method. The remainder of the paper is organized as follows: In the next section, the description and modeling of the full scale test rig are given. In Section 3, the stability and steady-state tracking errors for AMB system controlled by ILC controller with forgetting factor are derived. Design of the ILC controller and the simulation results are given in Section 4. Concluding remarks are shown in Section 5.

\section{The Full Scale Test Rig}

2.1. Overall Description of the System. Aiming at imitating and studying the control strategies and supercritical operation of turbomachine system, the full scale test rig is designed and established as a principle prototype rig in Division of Magnetic Bearing, INET, Tsinghua University. The whole system is installed in two places. To make debugging convenient, the control related components including controller facilities and power amplifiers are installed overground. To ensure the safety, the other components are installed in a concrete hole with $5 \mathrm{~m}$ in diameter and $10 \mathrm{~m}$ in height. The underground components of the system are shown in Figure 2. The high speed electric motor is equipped at top and connects with the turbine components by coupling. Several AMB units are settled along the rotor. 

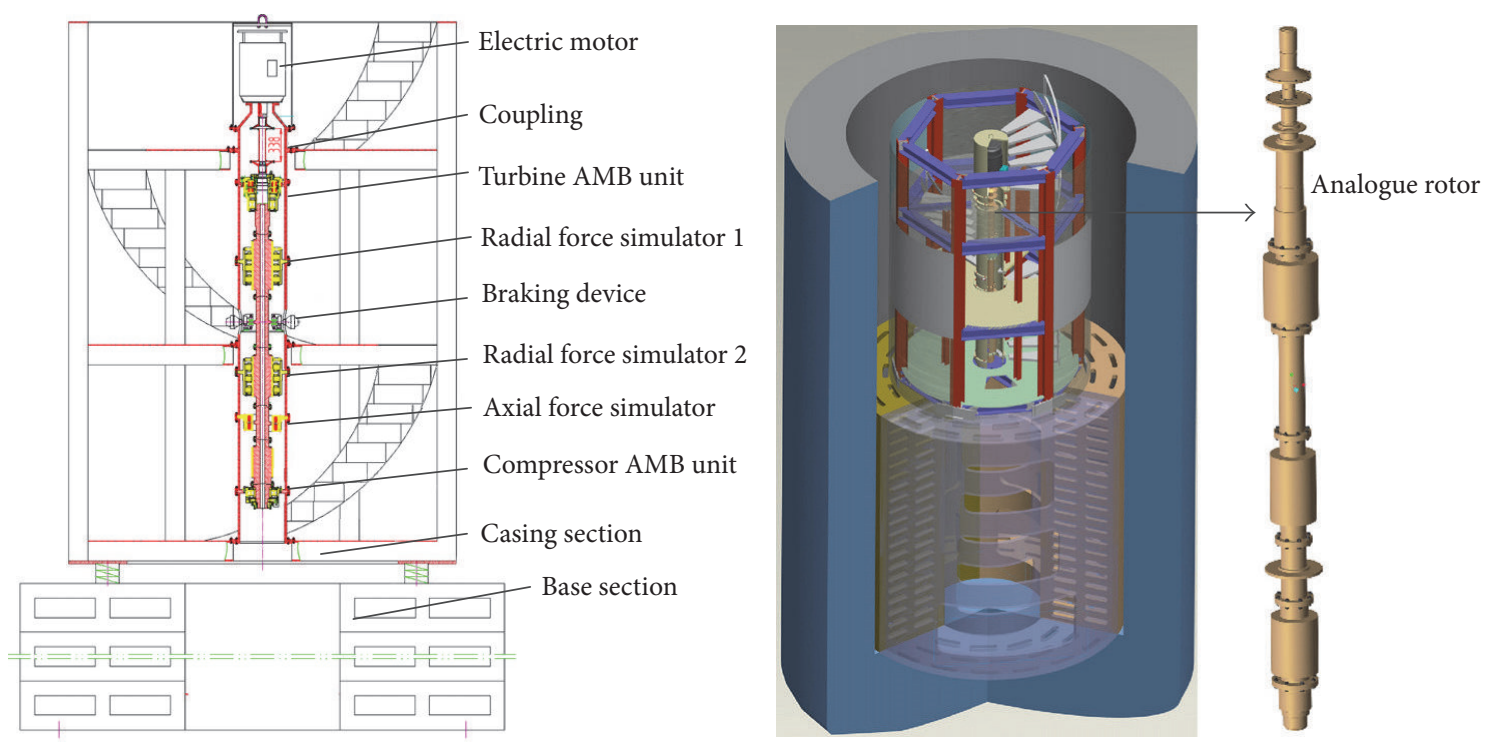

FIgURE 2: The underground parts of the full scale test rig.

The rotor is designed to have some characteristics which is similar to the turbine rotor. It is $3.55 \mathrm{~m}$ in length and $630 \mathrm{~kg}$ in weight which is almost the same as the actual turbine rotor. Moreover, the bending natural frequencies, the stiffness distribution, and the bending modals of the rotor are also close to those of the actual turbine rotor, such that the maximum error of the first two bending frequencies between the rotor and the actual one is less than 15\% [21]. As illustrated in Figure 3, there are two AMB assemblies located at the top and the bottom of the rotor, respectively, to support the rotor in radial directions. A thrust bearing is located at the top of the rotor to stabilize the rotor in axial direction. The displacement sensors are placed close to the AMBs.

In order to have deep insight into the rotor, rotor dynamic analysis is performed. The resulting free-free mode shape of the rotor is given in Figure 4. Based on this map, we can predict the vibrations of the rotor when the bending frequency nodes are excited. Additionally, the positions of the sensors and AMBs are also marked in the map which show the observability and controllability of the full scale test rig, intuitively. One conclusion can be made that the observability of the first bending mode, the second bending mode, and the third bending mode is good, because the sensors are in proper positions where they can detect enough information of the rotor displacements. Another conclusion is that the controllability of the third bending mode is insufficient, as the position of the AMB at the bottom is close to the node of the third bending mode. In that case, the vibration of the rotor cannot be controlled effectively when the third bending mode is excited.

An important feature for the rotor supported by AMBs is that the natural frequencies vary with the support stiffness. Thus, another important map for rotordynamics analysis is the critical speed map of the rotor. The map can be obtained by changing the support stiffness and the result is shown in Figure 5. It could be seen that the first bending frequency, the second bending frequency, and the third bending frequency are started at $50.2 \mathrm{~Hz}$ (3012 RPM), $147.9 \mathrm{~Hz}$ (8874 PRM), and $290.3 \mathrm{~Hz}$ (17418 PRM), respectively. It indicates that, before arriving the rated operation speed $15000 \mathrm{RPM}$, the rotor has to pass the second critical speed. The map is divided into three zones. In zone A where the support stiffness is much smaller than flexural rigidity of the material, the bending frequencies keep invariance while the rigid frequencies vary linearly in logarithmic coordinate. With the increasing of support stiffness, the magnitudes of the support stiffness and flexural rigidity of the material are at the same level; then, both rigid frequencies and flexible frequencies rise with the increase of support stiffness as shown in zone B. When the support stiffness is much larger than the flexural rigidity of the material, the natural frequencies will keep invariance as shown in zone C. Based on this map, we can predict the support stiffness at which the natural frequencies will be excited.

The rotor dynamic analysis results can help us have deep insight into the rotor characteristics, which will provide valuable information for controller design. Based on the analysis, a feedback PD controller is designed to stabilize the AMB system.

2.2. Modeling of the Closed-Loop AMB System. As mentioned in Section 2, the rotor of the full scale test rig is controlled by two AMB assemblies in radial directions. Therefore, if only the movements in radial directions are considered, the system is a Multi-Input and Multi-Output (MIMO) system with 4 inputs and 4 outputs. A diagram of the closed-loop AMB system is shown in Figure 6. For clarity, the main components of the system are marked in blue and all the components are modeled in the modeling process. To describe the MIMO system, all the components in the figure are presented by transfer function matrices and all the signals are vectors. 


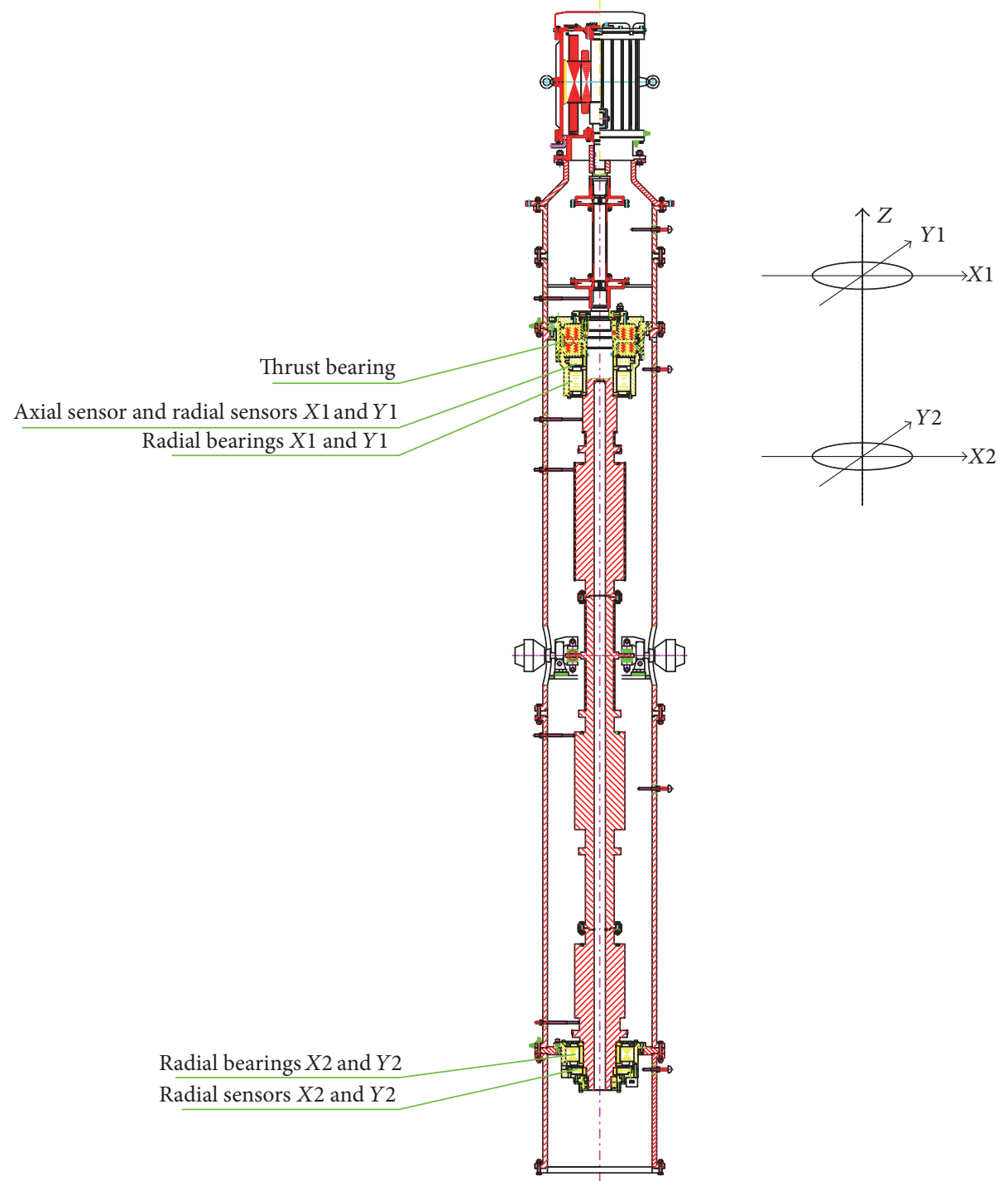

Figure 3: The structure of the full scale test rig.

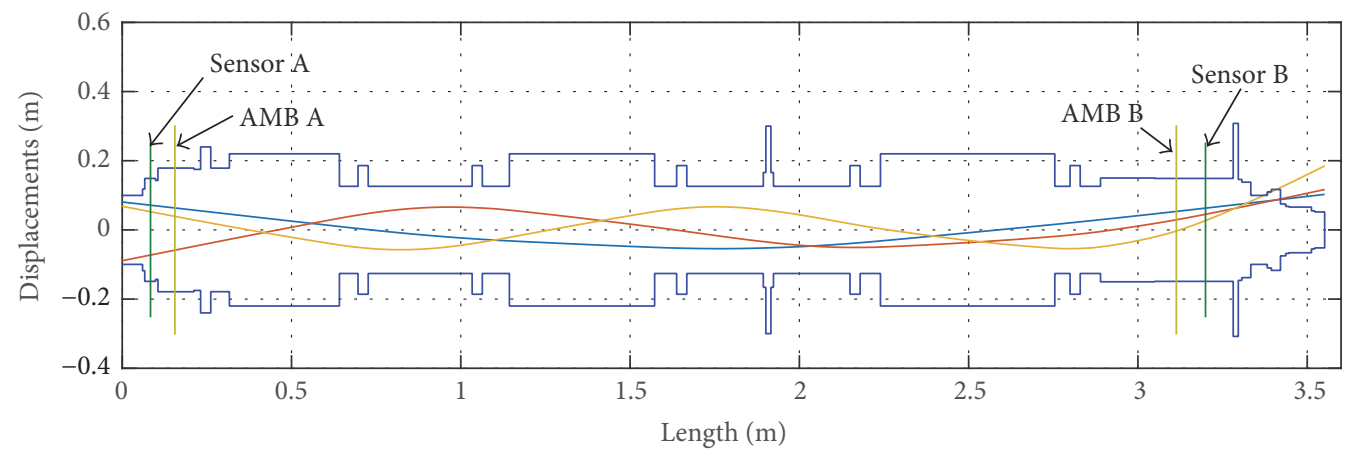

FIGURE 4: The undamped free-free mode shape of the rotor. 


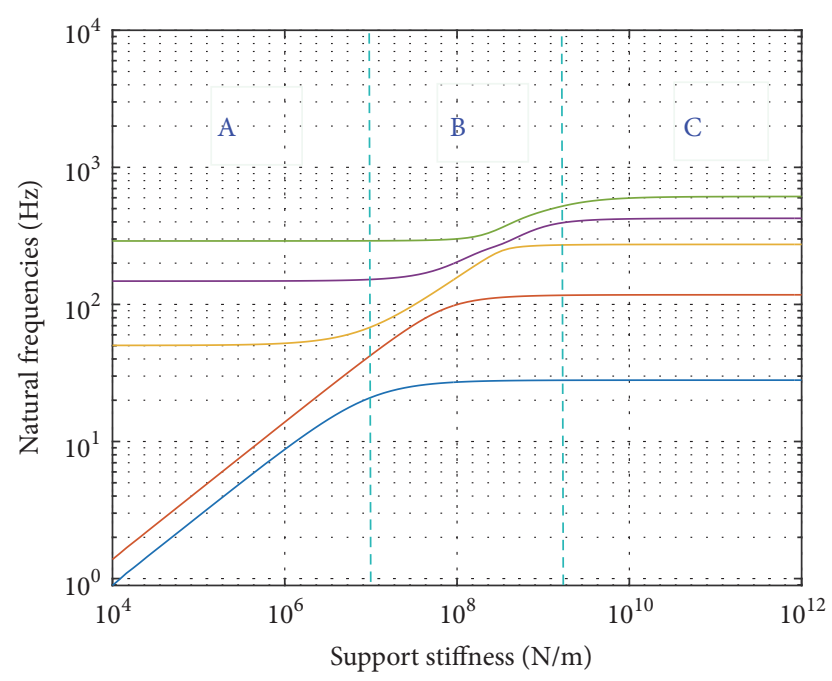

FIGURE 5: Nature frequencies in dependence of support stiffness.

The transfer function matrix of the rotor $\mathbf{R}(s)$ is a block diagonal matrix under two proper simplifications. One simplification is that the rotor is regarded as a rigid body. Another simplification is that the gyroscopic effect is neglected as the influence of gyroscopic effect for slender rotor is small. For the sensors and amplifiers, their dynamic characteristics are omitted in the modeling process as the bandwidth of the sensors and the amplifiers in practical applications are wide enough. It has been validated by applications that the omission leads to fairly good results [22]. The sensors and amplifiers are described by gain matrices $\mathbf{A}$ and $\mathbf{S}$, respectively. For the AMBs, the conventional linearized AMB model is adopted and it is written as

$$
\mathbf{F}_{m}(s)=\mathbf{K}_{i}(s) \mathbf{I}(s)-\mathbf{K}_{x}(s) \mathbf{X}(s),
$$

where $\mathbf{I}(s)$ is for the control current and $\mathbf{X}(s)$ is for rotor displacement. $\mathbf{K}_{i}(s)$ and $\mathbf{K}_{x}(s)$ are diagonal matrices with force current factors and force displacement factors as diagonal elements, respectively. It should be pointed out that the force displacement factor, also known as "negative stiffness," is negative which makes the open-loop AMB system unstable. Thus, to stabilize the system, decentralized PD controllers are involved and are described by a diagonal transfer function matrix $\mathbf{C}(z)$ with diagonal elements referring to discrete time transfer function of the PD controllers.

2.3. Unbalance Vibrations of the $A M B$ System. Unbalance vibrations are a result from the unbalance mass of the moving parts. Ideally, the mass center and geometrical center of an homogeneous rigid body are coincident; then, unbalance vibrations will not appear. However, in practice, the existence of the unbalance mass is unavoidable on account of the material's consistency, manufacturing error, rotor abrasion, and so forth. The unbalance mass will change mass distribution of the rotor and shift the mass center of the rotor away from its geometrical center. When the rotor is trying to rotate around its geometrical center, therefore, there will be a resultant centrifugal force acting on the rotor which will result in unbalance vibrations in rotor displacements. We assume that the initial phase of the resultant centrifugal force is $\theta$, the unbalance mass is $m$, and the eccentricity caused by unbalance mass is $e$. When a rotor is rotating with an angular speed $\omega$, then, the unbalance forces (the resultant centrifugal force) can be written as

$$
\begin{aligned}
& f_{i x}=m \omega^{2} e \cos (\omega t+\theta), \\
& f_{i y}=m \omega^{2} e \sin (\omega t+\theta) .
\end{aligned}
$$

Here, the subscript $i$ denotes the position along the rotor where the unbalance force acts. The subscripts $x$ and $y$ denote the two orthogonal directions in radial directions. Equation (2) shows that the unbalance effect will be sharp under conditions where the unbalance mass is large or under the other conditions where the angular speed is high. That is to say, the unbalance effect should be considered for the systems with large rotor imbalance or high operation angular speed. Thus, for the heavy rotating machinery with high speed like the turbine system, unbalance compensation technology should be involved. It should be emphasized that unbalance compensation is essentially a periodic disturbance rejection problem as the unbalance forces and the unbalance vibrations are periodic. In the next section, an unbalance compensation method for the AMB system is introduced.

\section{Frequency-Domain Iterative Learning Control Algorithm}

To attenuate the unbalance vibrations of the AMB system, an ILC controller is applied to modify the reference signal and the detailed structure is shown in Figure 7 [23]. Let $\mathbf{G}(s)$ be the Laplace transfer function matrix between the reference signal $\mathbf{r}(s)$ and output signal $\mathbf{y}(s), \mathbf{D}(s)$ be the Laplace transfer function matrix from the periodic disturbance signal $\mathbf{f}_{d}(s)$ to output signal $\mathbf{y}(s)$, and $\mathbf{C}(s)$ be the Laplace transfer function matrix of the feedback controller in continuous time obtained by adequate continuous approximation. Then, we have

$$
\begin{aligned}
\mathbf{G}(s) & =\left(\mathbf{I}+\mathbf{C}(s) \mathbf{A K}_{I}(s) \mathbf{R}_{\mathbf{K}}(s) \mathbf{R}(s) \mathbf{S}\right)^{-1} \mathbf{C}(s) \mathbf{A K}_{I}(s) \\
\cdot \mathbf{R}_{\mathbf{K}}(s) \mathbf{R}(s) \mathbf{S} & \\
\mathbf{D}(s) & =\left(\mathbf{I}+\mathbf{C}(s) \mathbf{A K}_{I}(s) \mathbf{R}_{\mathbf{K}}(s) \mathbf{R}(s) \mathbf{S}\right)^{-1} \mathbf{R}(s) \mathbf{S}
\end{aligned}
$$

with $\mathbf{R}_{\mathbf{K}}(s)=(\mathbf{I}-\mathbf{R}(s) \mathbf{K}(s))^{-1} \mathbf{R}(s)$. Here, I is an identity matrix with appropriate dimensions. The system response to reference signal and periodic disturbance can be derived as

$$
\mathbf{y}(s)=\mathbf{G}(s) \mathbf{r}(s)+\mathbf{D}(s) \mathbf{f}_{d}(s)
$$

As the disturbance signal is periodic, if the time interval of one iteration is equal to the period of the disturbance signal, (4) can be rewritten in iteration form

$$
\mathbf{y}_{k}(s)=\mathbf{G}(s) \mathbf{r}_{k}(s)+\mathbf{D}(s) \mathbf{f}_{d}(s) .
$$

Here, the subscript $k$ denotes the number of iterations and each iteration has the same time duration as the period of 


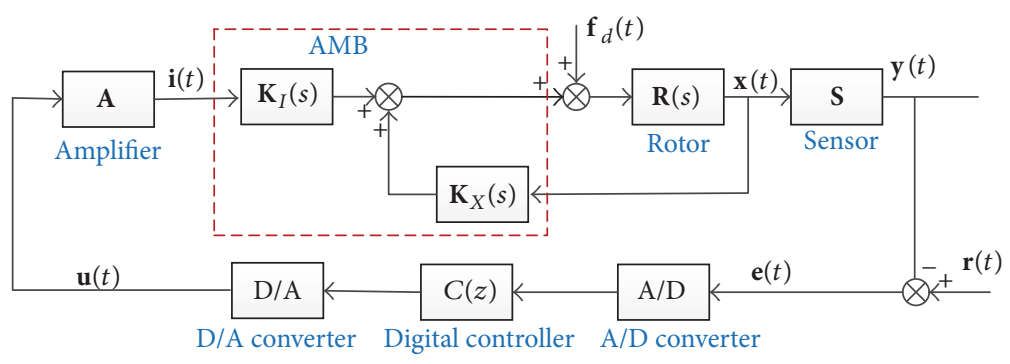

Figure 6: The closed-loop AMB system.

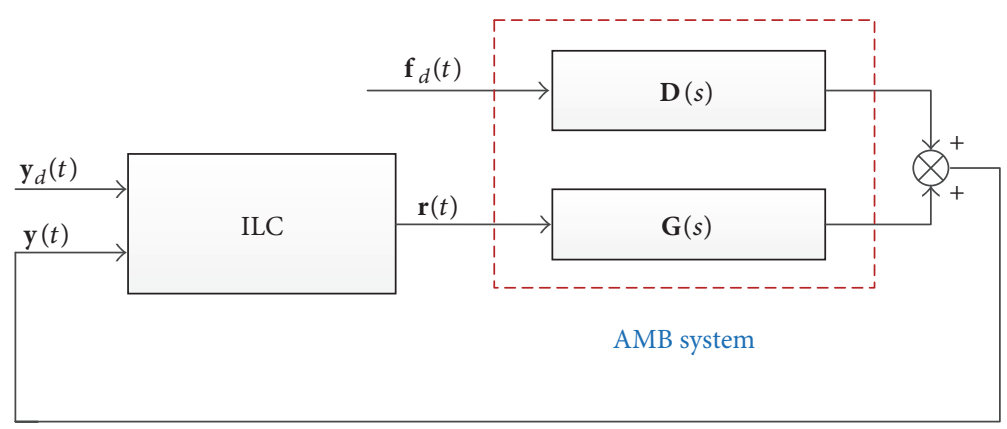

Figure 7: The AMB system with ILC.

the disturbance signal. Note that the system response to the periodic disturbance can be counteracted if we can find a reference signal

$$
\mathbf{r}_{k}(s)=-\mathbf{G}(s)^{-1} \mathbf{D}(s) \mathbf{f}_{d}(s) .
$$

But in practice, the reference signal cannot be obtained directly, since the magnitude and initial phase of the periodic signal $\mathbf{f}_{d}(s)$ are unknown. In the paper, the reference signal is updated, iteratively, based on the error signal and reference signal in previous iteration. Let us define the error signal as

$$
\mathbf{e}_{k}(t)=\mathbf{y}_{d}(t)-\mathbf{y}_{k}(t)
$$

where $\mathbf{y}_{d}(t)$ is the desired output signal. Taking Laplace transfer on both sides of (7) yields

$$
\mathbf{e}_{k}(s)=\mathbf{y}_{d}(s)-\mathbf{y}_{k}(s)
$$

When considering the initial errors, measurement noise, and fluctuations of the AMB system, the ILC controller with forgetting factor is adopted and the control signal updating law can be expressed as

$$
\mathbf{r}_{k+1}(s)=\mu \mathbf{r}_{k}(s)+\mathbf{L}(s) \mathbf{e}_{k}(s) .
$$

Here, $\mu \in(0,1]$ is a constant representing the forgetting factor. $\mathbf{L}(s)$ is an operator in frequency domain. Considering the error signal in $(k+1)$ th iteration, we have

$$
\begin{aligned}
\mathbf{e}_{k+1}(s)= & \mathbf{y}_{d}(s)-\mathbf{G}(s) \mathbf{r}_{k+1}(s)-\mathbf{D}(s) \mathbf{f}_{d}(s) \\
= & \mathbf{y}_{d}(s)-\mu \mathbf{G}(s) \mathbf{r}_{k}(s)-\mathbf{D}(s) \mathbf{f}_{d}(s) \\
& -\mathbf{G}(s) \mathbf{L}(s) \mathbf{e}_{k}(s) \\
= & (\mu \mathbf{I}-\mathbf{G}(s) \mathbf{L}(s)) \mathbf{e}_{k}(s) \\
& +(\mu-1)\left(\mathbf{D}(s) \mathbf{f}_{d}(s)-\mathbf{y}_{d}(s)\right) .
\end{aligned}
$$

For the AMB system, the desired system output is always set as $\mathbf{y}_{d}(s)=\mathbf{0}$; then (10) can be rewritten as

$$
\begin{aligned}
\mathbf{e}_{k+1}(s)= & (\mu \mathbf{I}-\mathbf{G}(s) \mathbf{L}(s)) \mathbf{e}_{k}(s) \\
& +(\mu-1) \mathbf{D}(s) \mathbf{f}_{d}(s)
\end{aligned}
$$

According to (11), we have

$$
\begin{aligned}
\mathbf{e}_{k+h}=( & \mu \mathbf{I}-\mathbf{G}(s) \mathbf{L}(s))^{k} \mathbf{e}_{h}(s)+(\mu-1) \\
& \cdot(\mathbf{I}-\mu \mathbf{I}+\mathbf{G}(s) \mathbf{L}(s))^{-1} \\
& \cdot\left(\mathbf{I}-(\mu \mathbf{I}-\mathbf{G}(s) \mathbf{L}(s))^{k}\right) \mathbf{D}(s) \mathbf{f}_{d}(s) .
\end{aligned}
$$



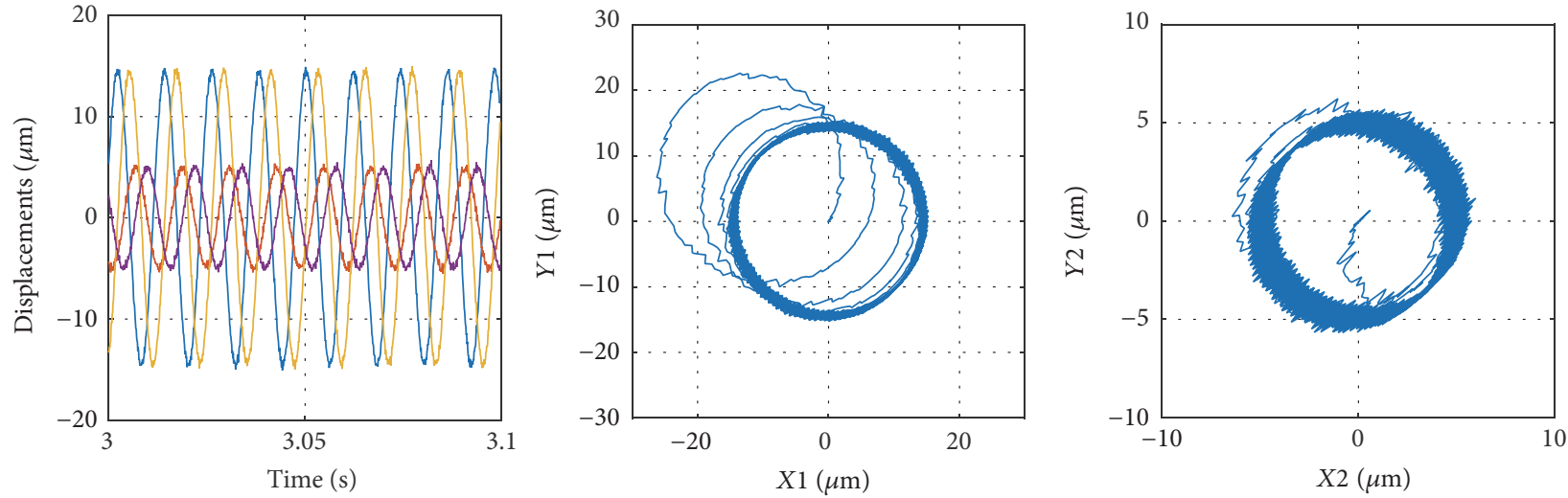

FIGURE 8: Unbalance responses of the closed-loop AMB system.

Taking norm on both sides of (12) yields

$$
\begin{aligned}
\left\|\mathbf{e}_{k+h}\right\| \leq & \left\|(\mu \mathbf{I}-\mathbf{G}(s) \mathbf{L}(s))^{k}\right\|\left\|\mathbf{e}_{h}(s)\right\|+(1-\mu) \\
& \cdot\left\|(\mathbf{I}-\mu \mathbf{I}+\mathbf{G}(s) \mathbf{L}(s))^{-1}\right\|\left\|\mathbf{D}(s) \mathbf{f}_{d}(s)\right\| \\
+ & (1-\mu)\left\|(\mathbf{I}-\mu \mathbf{I}+\mathbf{G}(s) \mathbf{L}(s))^{-1}\right\| \\
& \cdot\left\|(\mu \mathbf{I}-\mathbf{G}(s) \mathbf{L}(s))^{k}\right\|\left\|\mathbf{D}(s) \mathbf{f}_{d}(s)\right\| .
\end{aligned}
$$

Note that if $\|(\mu \mathbf{I}-\mathbf{G}(s) \mathbf{L}(s))\|<1$, then

$$
\begin{aligned}
& \lim _{k \rightarrow \infty}\left\|\mathbf{e}_{k+h}\right\| \\
& \quad \leq(1-\mu)\left\|(\mathbf{I}-\mu \mathbf{I}+\mathbf{G}(s) \mathbf{L}(s))^{-1}\right\|\left\|\mathbf{D}(s) \mathbf{f}_{d}(s)\right\| .
\end{aligned}
$$

Equations (13) and (14) indicate that the system equation (4) controlled by (9) is stable if

$$
\|(\mu \mathbf{I}-\mathbf{G}(s) \mathbf{L}(s))\|<1 .
$$

In order to facilitate the description, hereafter, the matrix $\mu \mathbf{I}-$ $\mathbf{G}(s) \mathbf{L}(s)$ is named as "stability matrix."

Remark 1. The original D-type ILC controller proposed by Arimoto is a special case of (9) in which $\mu=1$. In that case, freedom from noise, repeatability of initialization, and invariance of system dynamics are assumed, and the error is converged to zero as the number of iterations goes to infinity.

Remark 2. It is desired to point out that the system stability does not imply that the error when $k \rightarrow \infty$ is smaller than what is achieved without ILC control. Shown in (14) is that the steady-state error is governed by $(1-\mu) \|(\mathbf{I}-\mu \mathbf{I}+$ $\mathbf{G}(s) \mathbf{L}(s))^{-1}\|\| \mathbf{D}(s) \mathbf{f}_{d}(s) \|$.

From the above analysis, the design objectives of ILC controller could be concluded as follows: finding two parameter matrices, $\mu$ and $\mathbf{L}(s)$, such that

(1) (15) is satisfied which ensures the stability of the AMB system controlled by ILC controller,

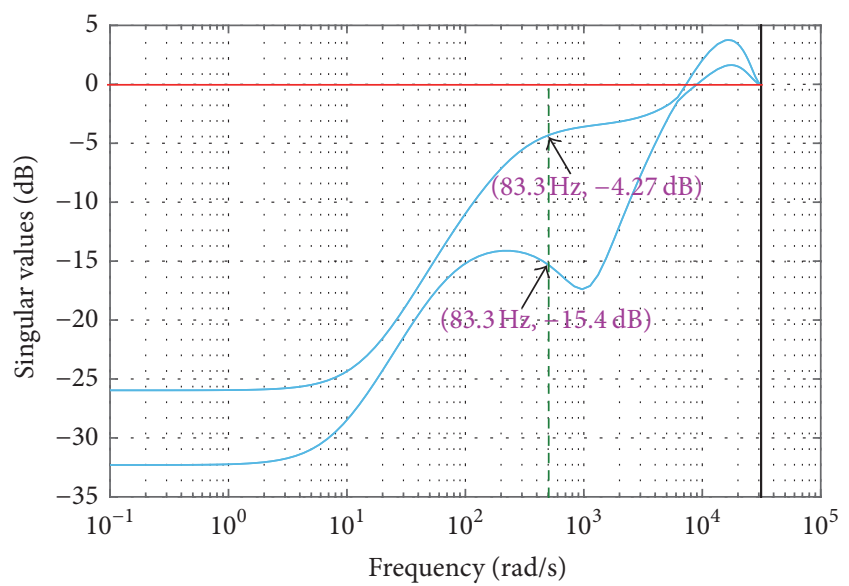

FIGURE 9: Singular values of the stability matrix.

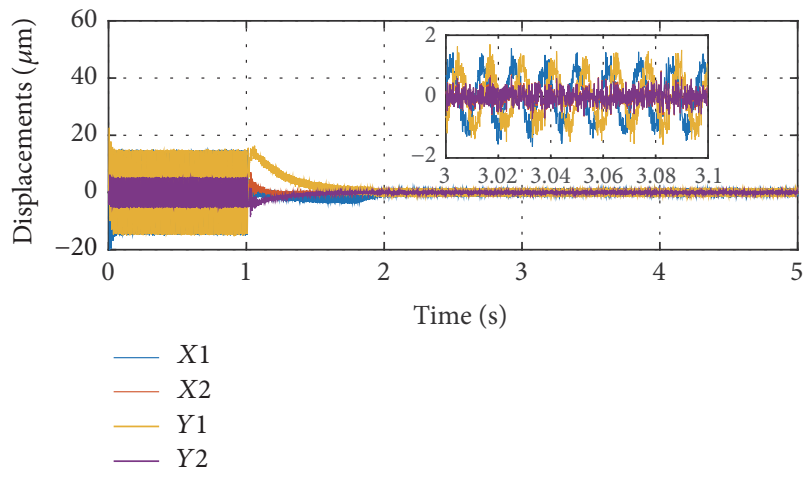

FIGURE 10: Shaft displacements in radial directions.

(2)

$$
(1-\mu)\left\|(\mathbf{I}-\mu \mathbf{I}+\mathbf{G}(s) \mathbf{L}(s))^{-1}\right\|<1
$$

which ensures that the unbalance vibrations are attenuated to some extent.

For the single-input and single-output system, the Nyquist frequency plot can be applied to analyze the stability and 

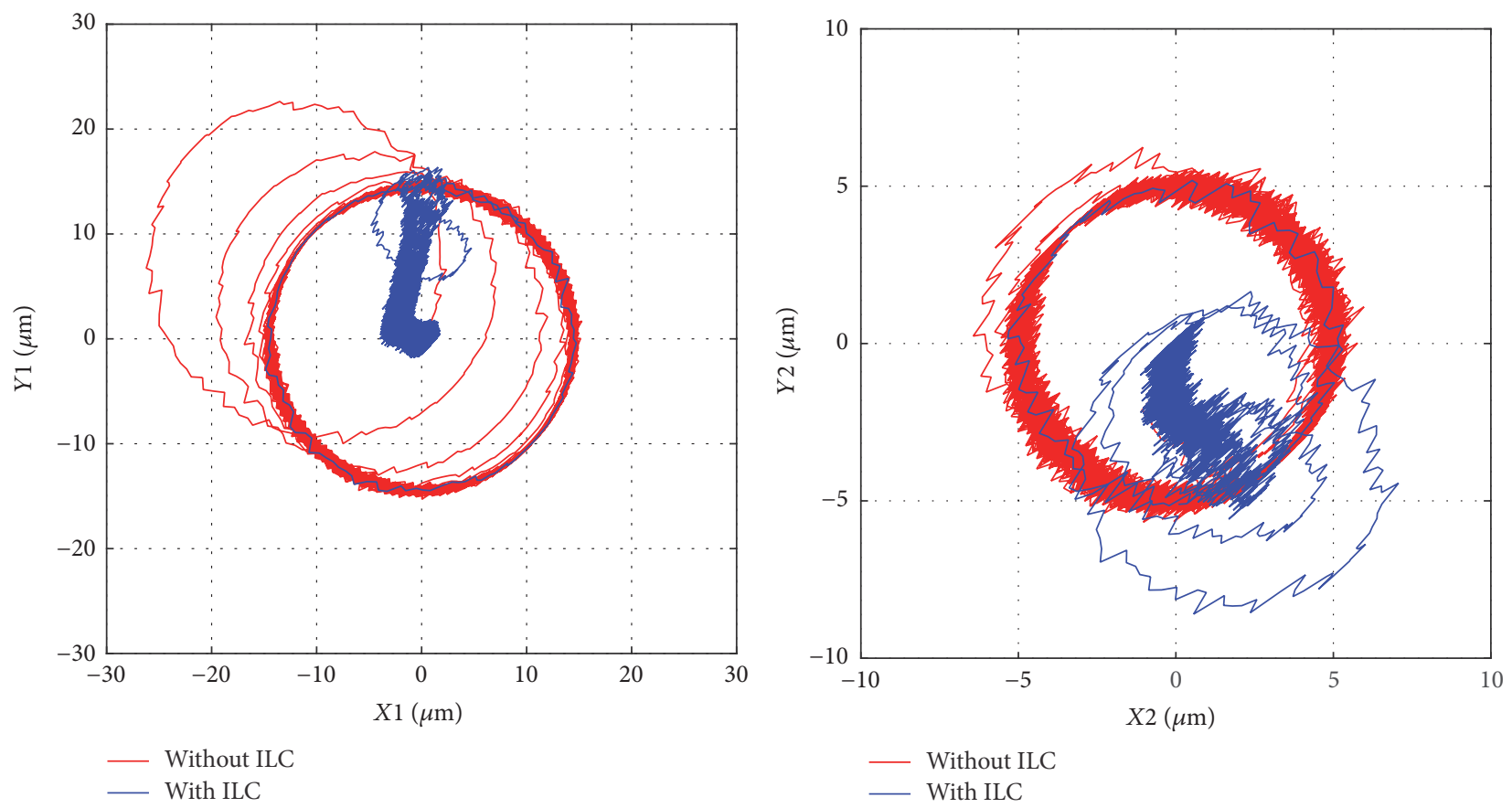

FIgURE 11: Orbits of the center of the shaft.

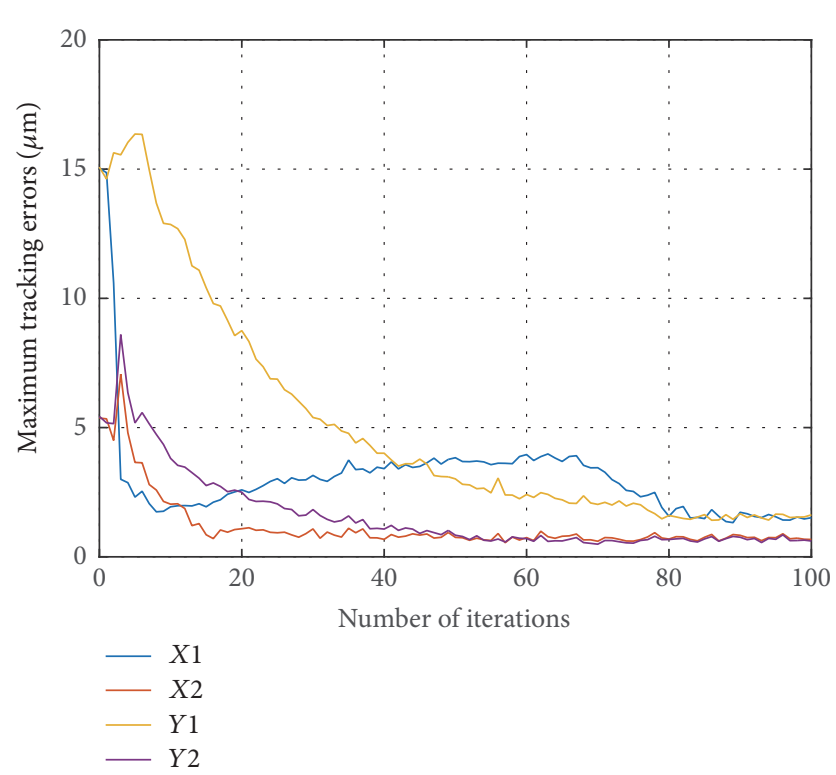

FIGURE 12: The maximum tracking errors versus number of iterations.

steady-state error. However, it is difficult to extend this method to MIMO system. Here, the singular value analysis method in robust theory is introduced. With the assumption that the matrix norm mentioned in the paper is $\infty$ norm, then (15) and (16) can be expressed as [24]

$$
\begin{aligned}
& \bar{\sigma}\{\mu \mathbf{I}-\mathbf{G}(s) \mathbf{L}(s)\}<1, \\
& (1-\mu) \bar{\sigma}\left\{(\mathbf{I}-\mu \mathbf{I}+\mathbf{G}(s) \mathbf{L}(s))^{-1}\right\}<1 .
\end{aligned}
$$

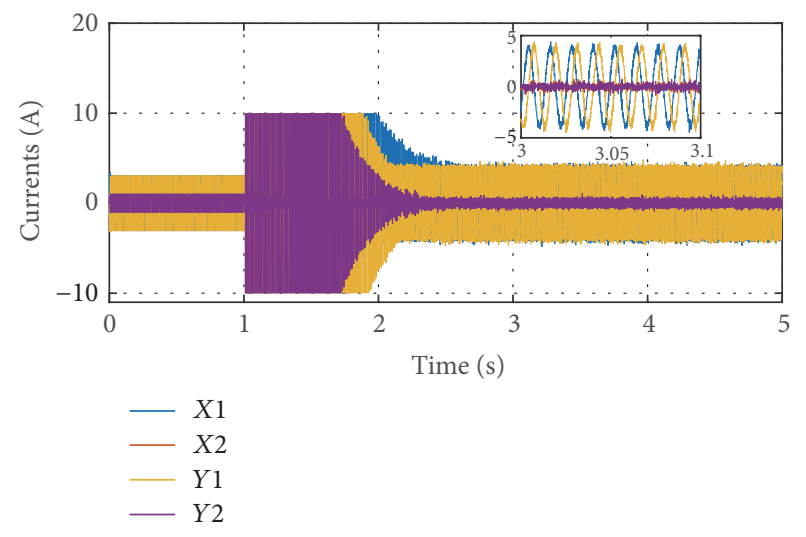

FIGURE 13: Control currents in radial directions.

Here $\bar{\sigma}$ denotes the maximum singular value of the matrix. Unbalance compensation for AMB system is inherently to attenuate the synchronous vibration in rotor displacements; thus, Fourier method is used to detect the synchronous component which is to be attenuated by ILC control. Thus, for continuous time system, the design objectives can be expressed as

$$
\begin{aligned}
& \bar{\sigma}\left\{\mu \mathbf{I}-\mathbf{G}\left(j \omega_{0}\right) \mathbf{L}\left(j \omega_{0}\right)\right\}<1, \\
& (1-\mu) \bar{\sigma}\left\{\left(\mathbf{I}-\mu \mathbf{I}+\mathbf{G}\left(j \omega_{0}\right) \mathbf{L}\left(j \omega_{0}\right)\right)^{-1}\right\}<1 .
\end{aligned}
$$

However, for discrete time system, it is [25]

$$
\begin{aligned}
\bar{\sigma}\left\{\mu \mathbf{I}-\mathbf{G}\left(e^{j \omega_{0} T_{s}}\right) \mathbf{L}\left(e^{j \omega_{0} T_{s}}\right)\right\} & <1, \\
(1-\mu) \bar{\sigma}\left\{\left(\mathbf{I}-\mu \mathbf{I}+\mathbf{G}\left(e^{j \omega_{0} T_{s}}\right) \mathbf{L}\left(e^{j \omega_{0} T_{s}}\right)\right)^{-1}\right\} & <1 .
\end{aligned}
$$



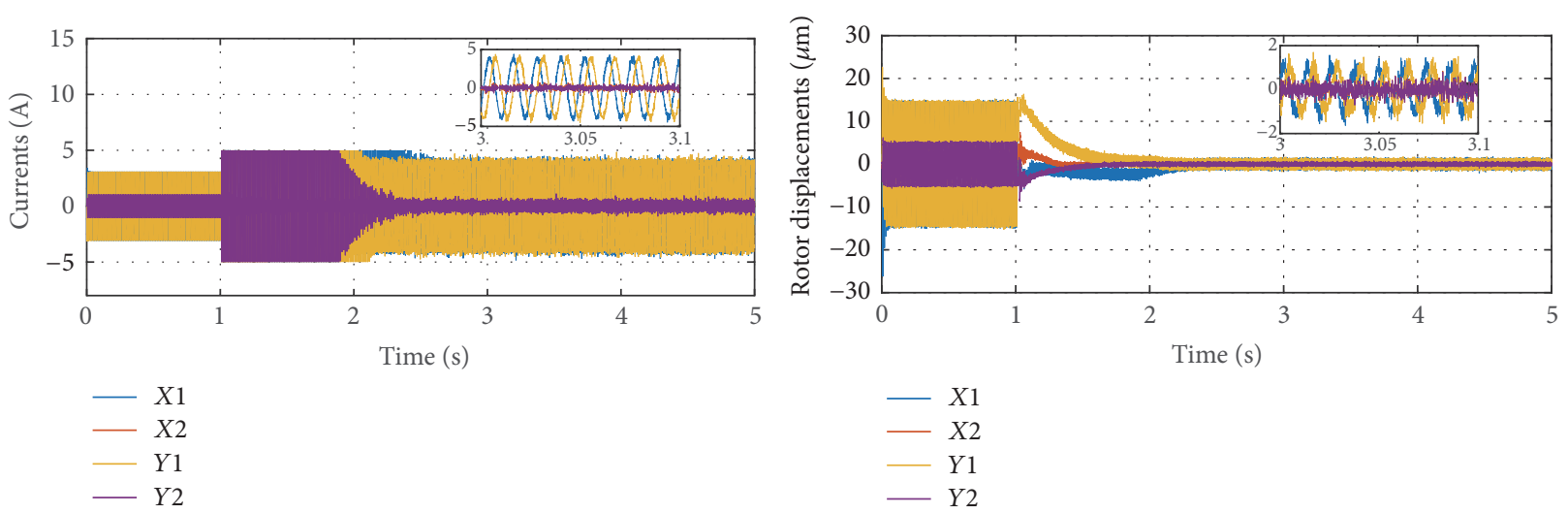

FIGURE 14: Control currents and rotor displacements in radial directions: saturation current is $5 \mathrm{~A}$.
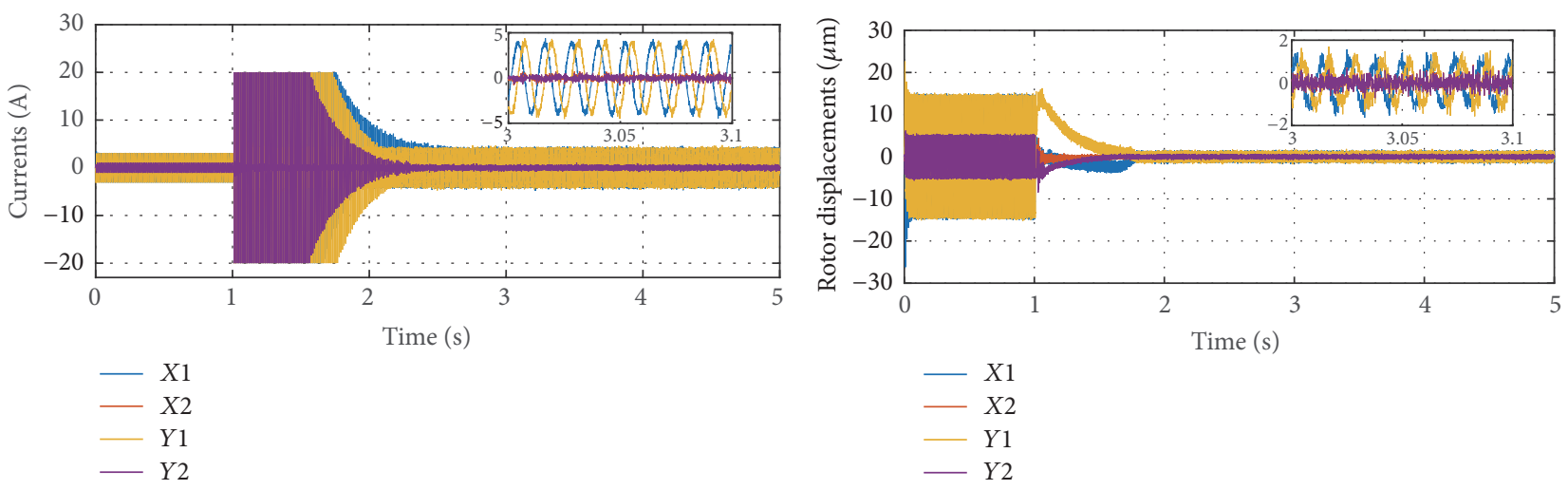

FIGURE 15: Control currents and rotor displacements in radial directions: saturation current is $20 \mathrm{~A}$.

Here, $\omega_{0}$ is the frequency of the unbalance vibrations to be attenuated and $T_{s}$ is the sample time. For specific application, the design objectives in singular form could be further simplified. In addition, the existing tools for frequencydomain analysis can be applied, directly, in ILC controller designing.

\section{Simulation Results}

In this section, the ILC controller with forgetting factor, as recalled in Section 3, is applied to the full scale test rig described in Section 2 to attenuate unbalance vibrations. The simulation studies are carried out based on the simulation model of the entire AMB system derived in Section 2 and the measurement noise which is modeled by white noise with zero-value is also included.

To model the unbalance vibrations, in the simulation, additional sines and cosines forces of $1000 \mathrm{~N}$ with initial phase of $0.3 \pi$, intentionally, are added to the radial AMB at one end. Simulation studies are carried out under the conditions where operation speed is initially fixed at 5000 RPM $(83.3 \mathrm{~Hz})$ and system sample time $T_{s}$ is set as $10^{-4} \mathrm{~s}$. Shown in Figure 8 is some simulation results including the rotor displacements in four radial directions and the orbits of the center of the shaft with respect to the geometrical centers of the magnetic bearings in each end. As estimated, when the system is only controlled by discrete time PD controllers, periodic vibrations appear in the rotor displacements. The simulation results show that the maximum amplitude of periodic vibrations, in this case, is $15 \mu \mathrm{m}$. Generation of this phenomenon is derived in [19]. As they reported, this phenomenon will appear when the initial phase of the unbalance force is not $\pi / 2+k \pi, k=0,1,2, \ldots$.

To reduce the periodic vibrations in the rotor displacements and increase the rotating accuracy of the rotor, an ILC controller is applied to modify the reference signal of the closed-loop AMB system. As the ILC controller is naturally a digital one, adequate discrete approximations are used to transfer the closed-loop AMB system into discrete time. Based on the design objectives mentioned in Section 4, an ILC controller in which $\mu=0.95$ and the diagonal elements of the operator in discrete time domain are $0.7+0.001(z-$ $1) / T_{s} z$ is designed. The stability of the system controlled by the designed ILC controller is checked by the singular values of stability matrix. As shown in Figure 9, under the condition where the operation speed is $5000 \mathrm{RPM}(83.3 \mathrm{~Hz})$, the maximum singular value of stability matrix is about $-4.27 \mathrm{~dB}$. That is to say, the system is stable as the first equation in (17) is satisfied.

In the simulation, the rotation period of rotor is fixed at $0.012 \mathrm{~s}(5000 \mathrm{RPM})$ and sample time is $10^{-4} \mathrm{~s}$. Thus there are 120 sample points in one iteration. Initially, the system 

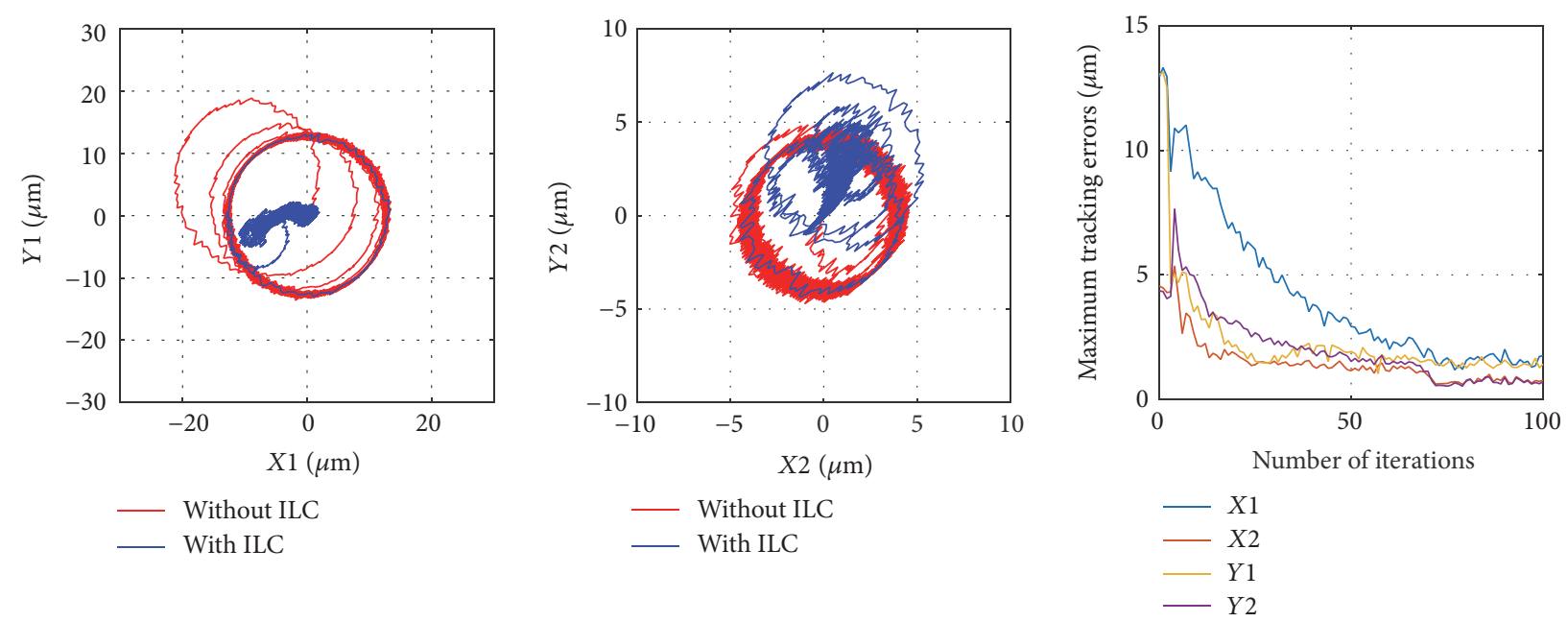

(a) The rotor operation speed is $4000 \mathrm{RPM}$
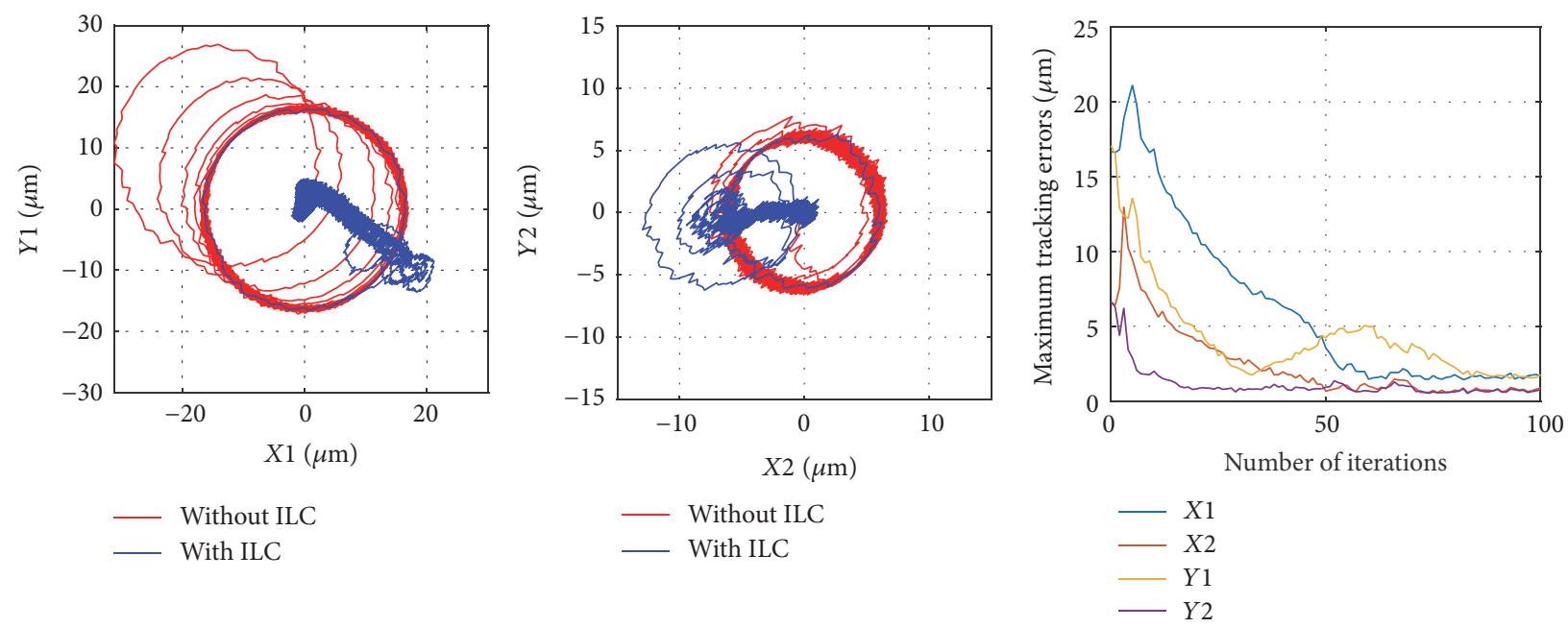

(b) The rotor operation speed is $6000 \mathrm{RPM}$

FIGURE 16: Orbits of the center of the shaft and the maximum tracking errors versus number of iterations with different rotor operation speeds.

is only controlled by the feedback PD controller, and the operation speed of 5000 PRM is obtained in safe conditions. The ILC controller for attenuation of unbalance vibration is activated at $1 \mathrm{~s}$. The simulation results are shown in Figures 10-13. Shown in Figure 10 are the shaft displacements in four radial directions. Note that the vibrations of the rotor increase slightly during the initial learning period and then decrease quickly up to the steady-state errors. As expected, the vibrations are attenuated, remarkably.

The orbits of the center of the shaft (with respect to the geometrical centers of the magnetic bearings) are shown in Figure 11, in which the orbits with and without ILC controller are colored in blue and red, respectively. It could be seen that the orbits of the center of the shaft with ILC controller are much smaller than what is achieved without ILC controller.

The convergence process in terms of maximum tracking error in each iteration at radial directions is shown in Figure 12. It can be observed that the tracking errors increase in initial iteration periods and then decrease quickly, finally reaching nonzero steady-state tracking errors. The results match with the shaft displacements in radial directions. It should be pointed out that the steady-state tracking errors are the result of a combination of measurement noise, initial errors, and the forgetting factor.

The control currents in radial directions are shown in Figure 13. Note that the amplitudes of the currents are abruptly enlarged at $1 \mathrm{~s}$ and even lead to saturation of power amplifier (in this case, the saturation current of the power amplifier is $10 \mathrm{~A})$. This phenomenon indicates that large control forces are required at initial iterations. As we all know, saturation of power amplifier should be avoided as it could reduce the system performances and even lead to failure. To further study this phenomenon, simulation studies with different saturation currents are carried out. Shown in Figures 14 and 15 are some simulation results which indicate that amplitude limiting on control currents only affects convergence speed of 

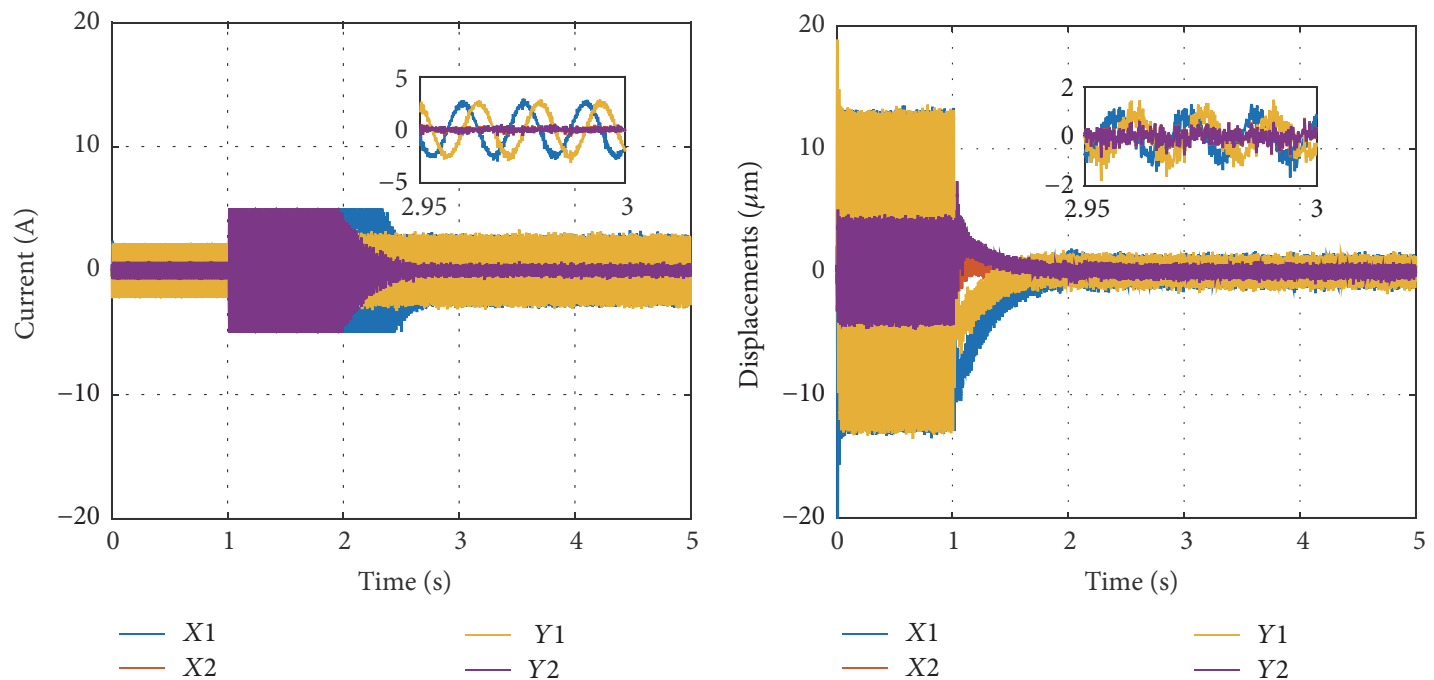

(a) The saturation current is $5 \mathrm{~A}$
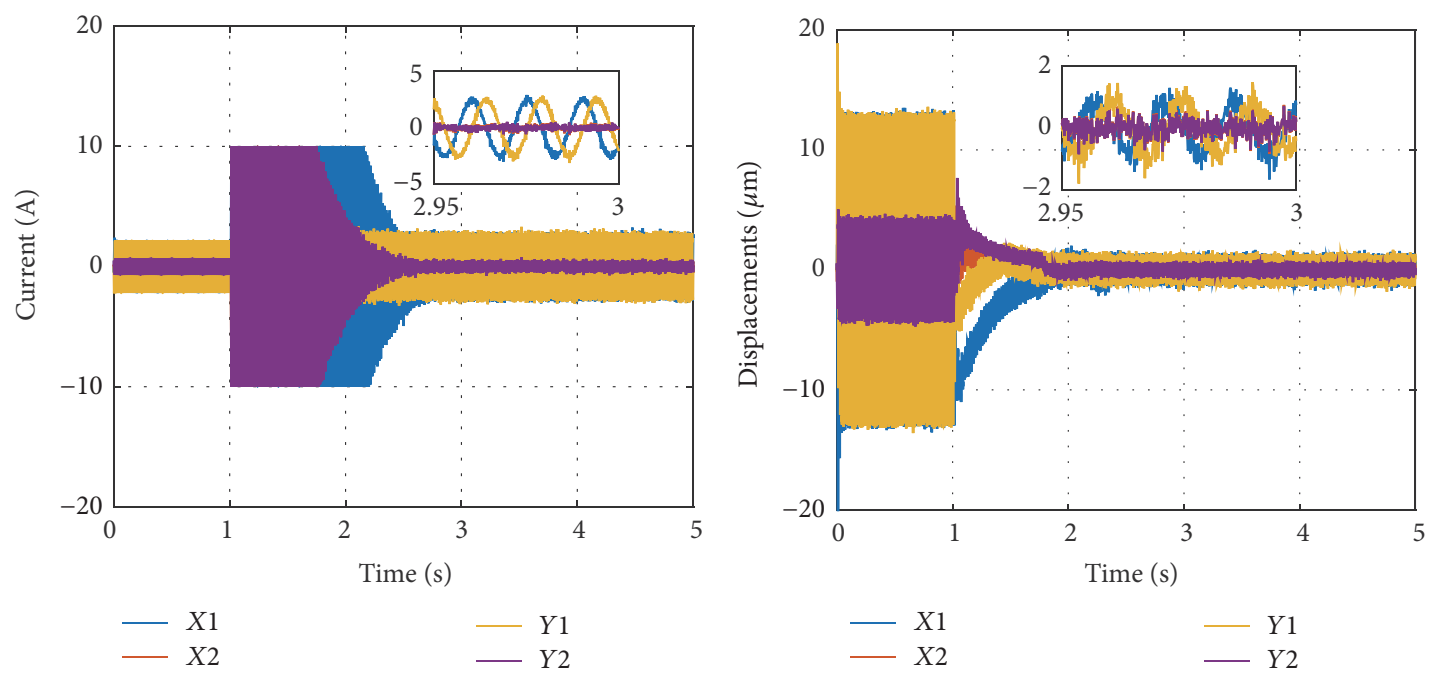

(b) The saturation current is $10 \mathrm{~A}$
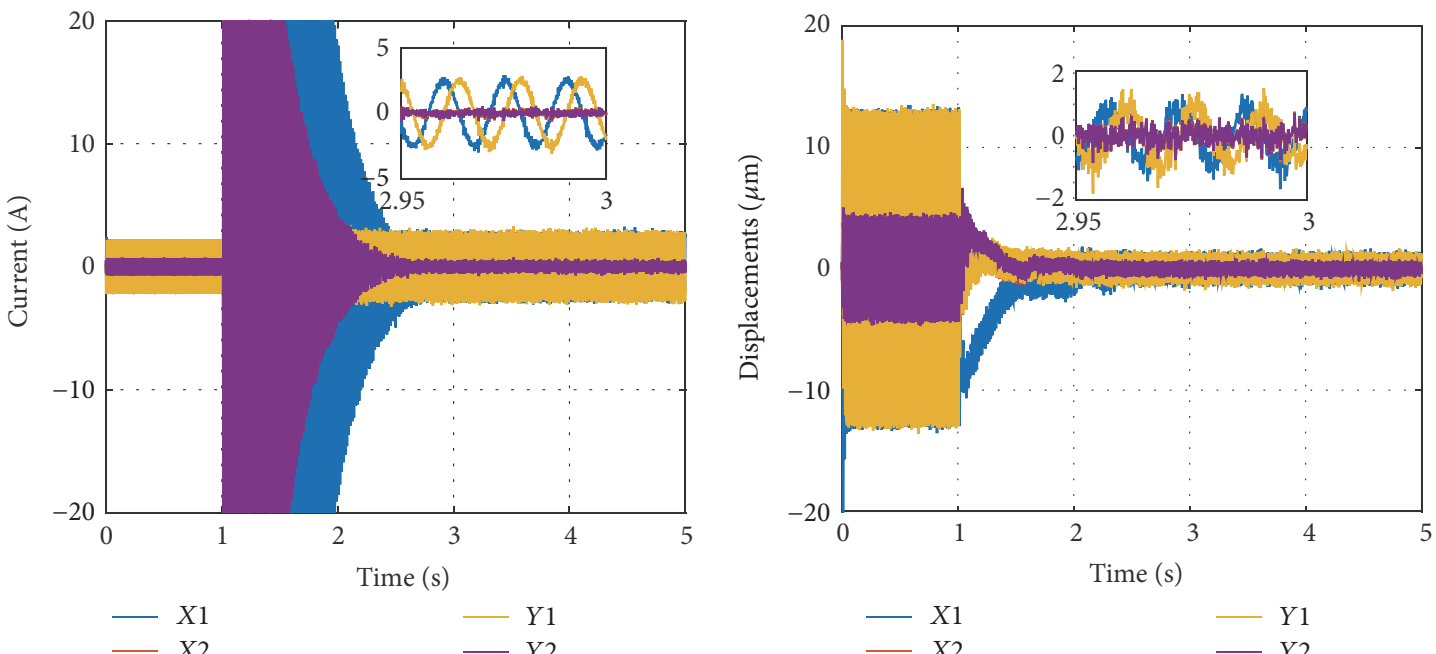

(c) The saturation current is $20 \mathrm{~A}$

FIGURE 17: Control currents and rotor displacements in radial directions with different power amplifier saturation currents: the rotor operation speed is 4000 RPM. 

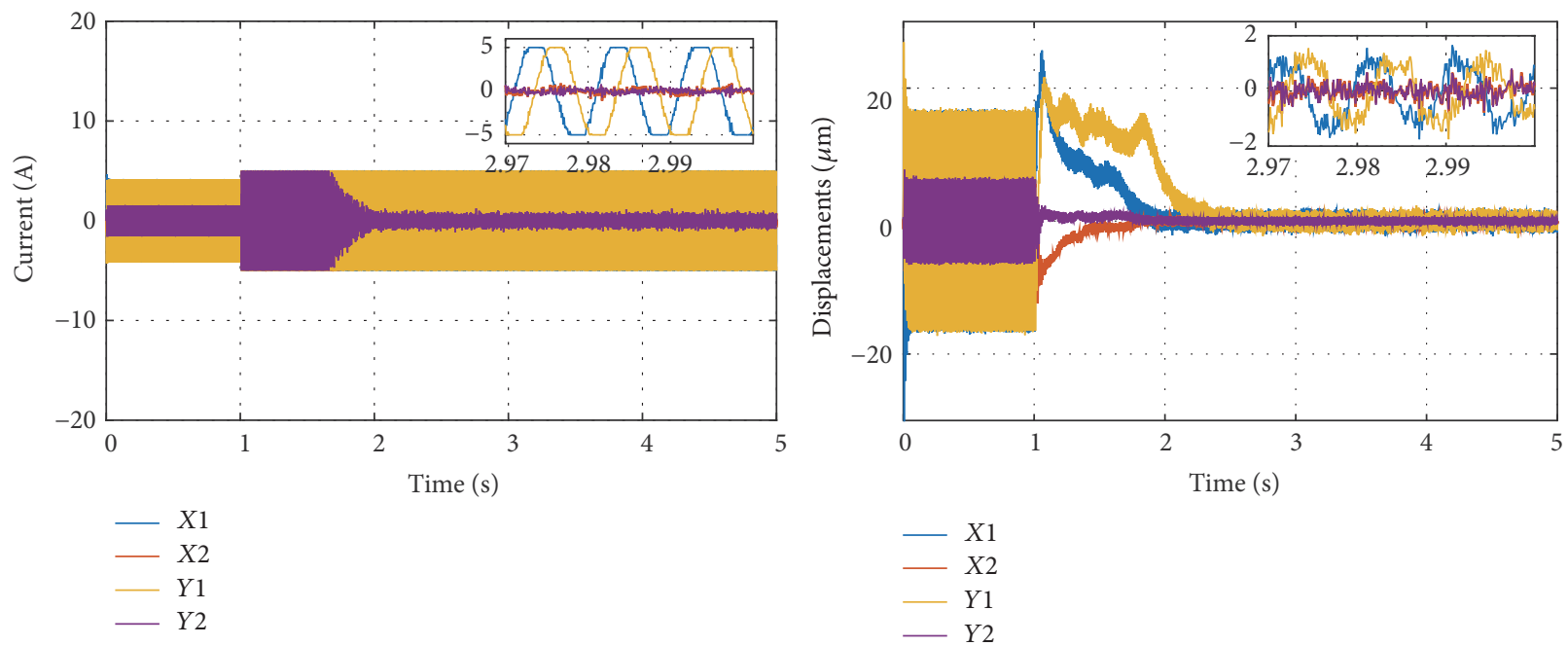

(a) The saturation current is $5 \mathrm{~A}$
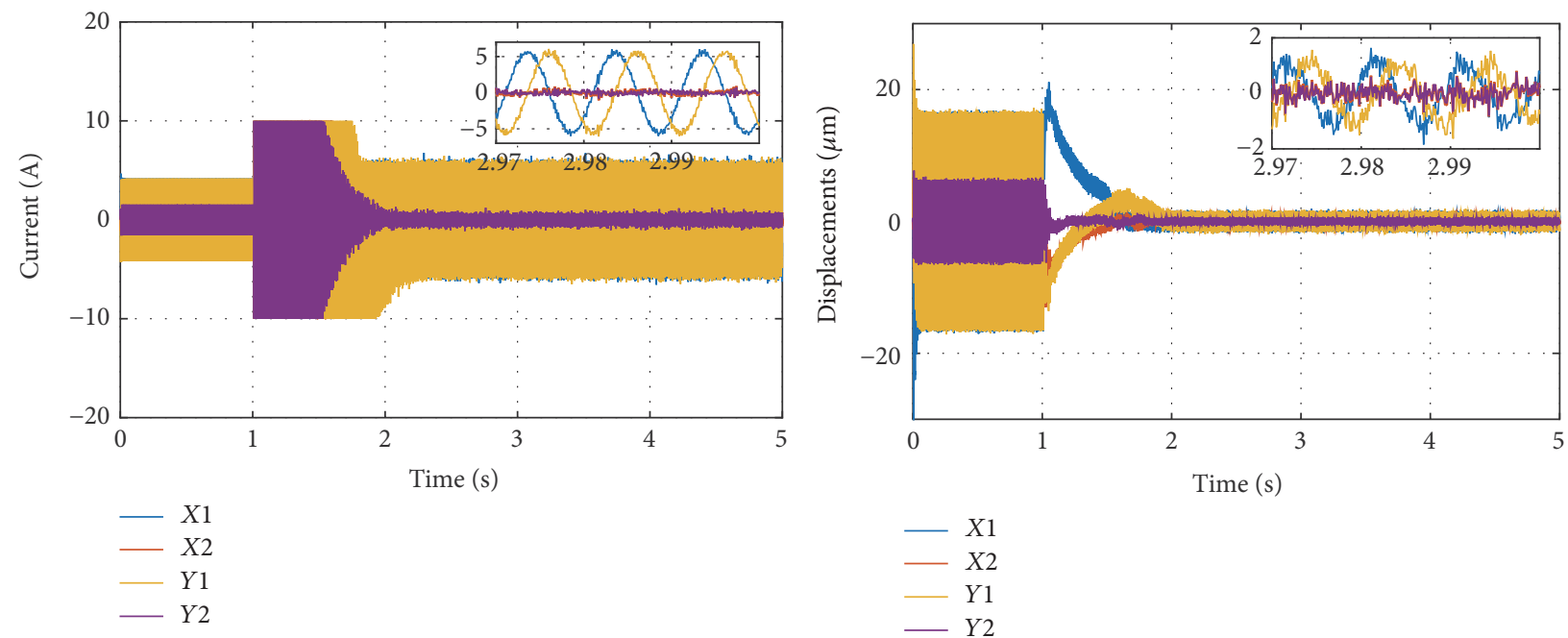

(b) The saturation current is $10 \mathrm{~A}$
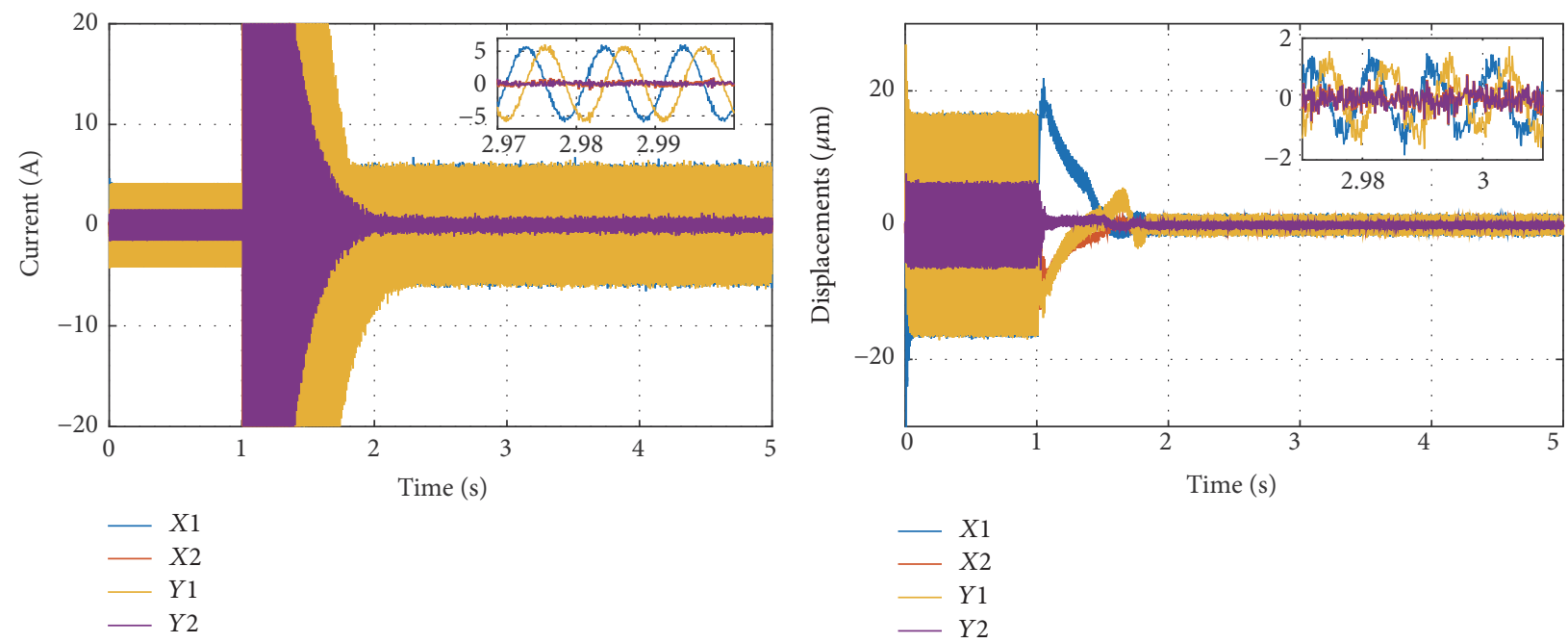

(c) The saturation current is $20 \mathrm{~A}$

FIGURE 18: Control currents and rotor displacements in radial directions with different power amplifier saturation currents: the rotor operation speed is $6000 \mathrm{RPM}$. 


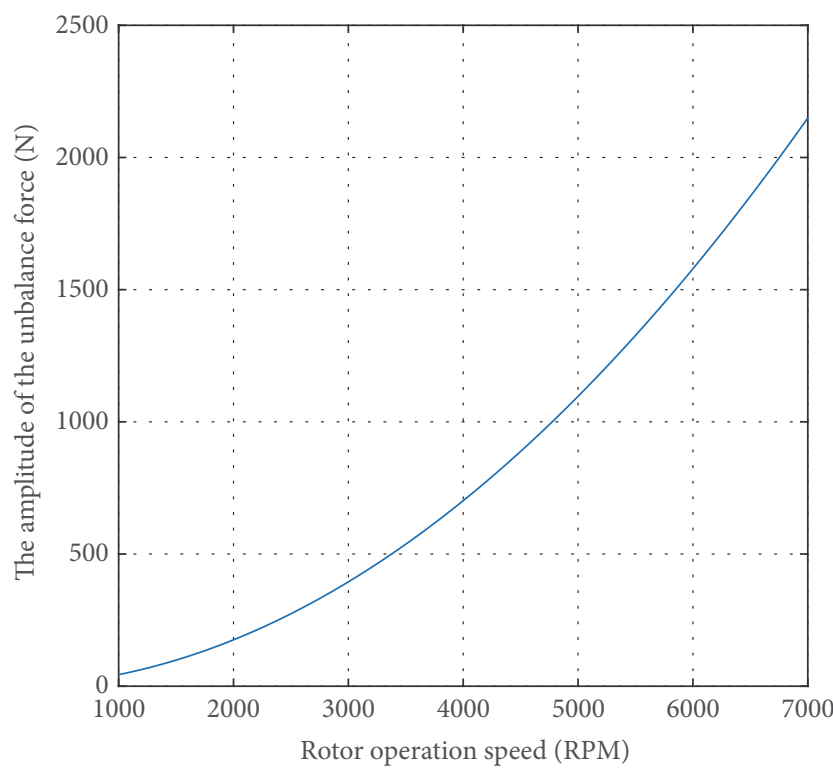

FIgURE 19: The amplitude of the unbalance force acts on the rotor.

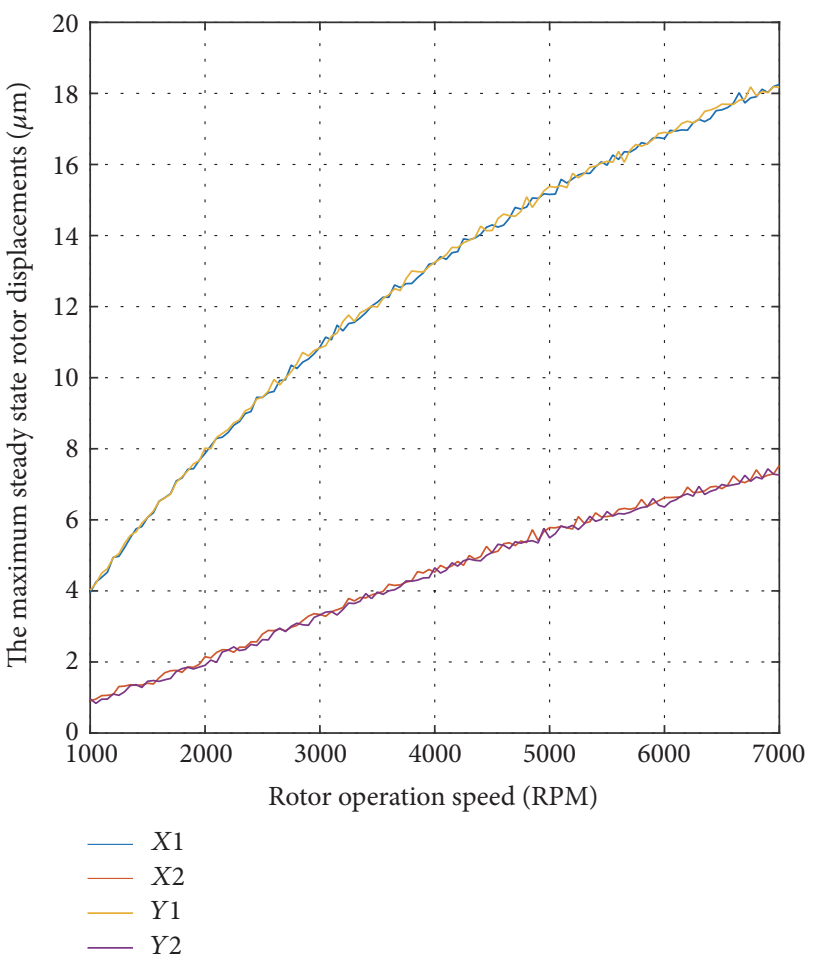

Figure 20: The maximum steady-state rotor displacements without unbalance control.

the rotor displacements, while the stability of the system and steady-state control currents keeps invariant. Thus it could be concluded that saturation of the power amplifier can be avoided by adding amplitude limiting with low thresholds without affecting system stability.

To confirm the conclusions made above, simulations under the conditions where the rotor operation speed is 4000 RPM and 6000 RPM, respectively, are carried out. As

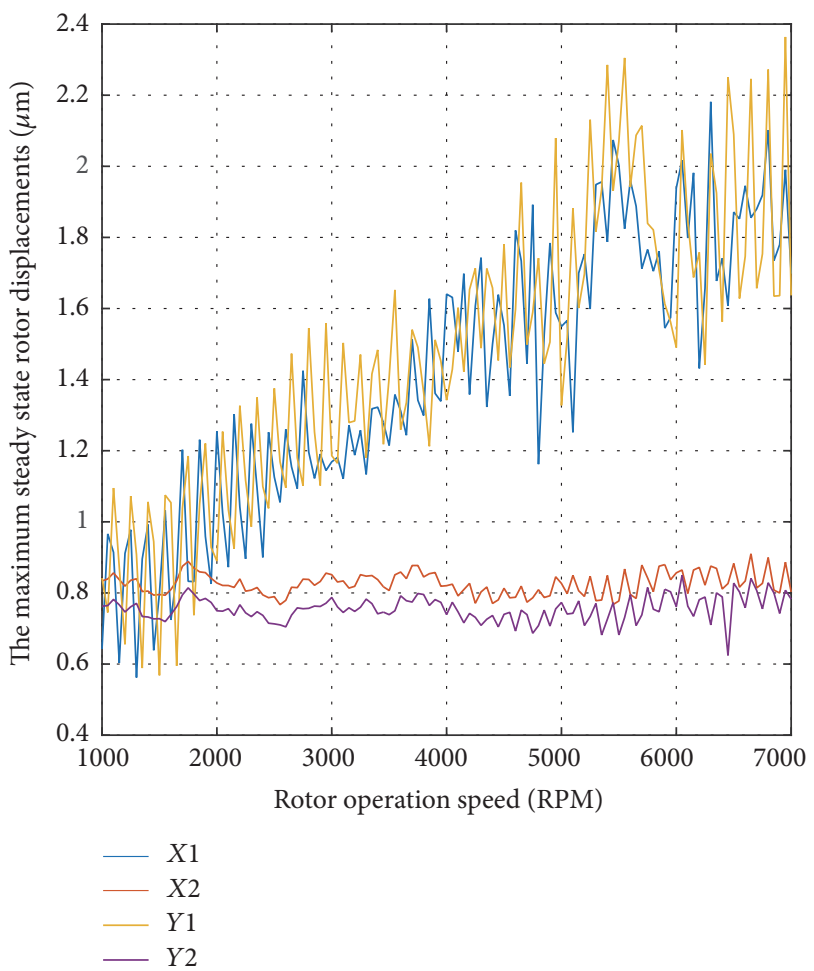

FIGURE 21: The maximum steady-state rotor displacements with ILC control.

shown in Figure 9, the singular values of the stability matrix under both rotor operation speeds are below $0 \mathrm{~dB}$ which ensure the stability of the system with ILC controllers. The simulation results are shown in Figures 16-18. Shown in Figure 16 are the orbits of the center of the shaft and the maximum tracking errors versus number of iterations when the rotor operation speed is $4000 \mathrm{RPM}$ and $6000 \mathrm{RPM}$. It is clear that, in both cases, the unbalance vibrations are effectively attenuated. Figures 17 and 18 show the influence of power amplifier saturation current on control currents and rotor displacements. As predicted, the saturation current only has influence on the convergency speed but has no significant effect on the convergency of the system and the steady-state control current and rotor displacements. The observations are the same as what is observed when the rotor operation speed is $5000 \mathrm{RPM}$.

To further study the effectiveness of the proposed method, unbalance compensation for AMB system with different operation speed is studied. The operation speed is initially $1000 \mathrm{RPM}$, then increases with a step of $50 \mathrm{RPM}$, and stops with 7000 RPM. The unbalance force acting on the rotor, the maximum steady-state rotor displacements without unbalance control, and the maximum steady-state rotor displacements with ILC are shown in Figures 19, 20, and 21 , respectively. To model the unbalance force results from imbalance mass of the rotor, two forces whose amplitude is proportional to the square of rotor operation speed are intentionally applied to the $X 1$ and $X 2$ directions of the rotor as shown in Figure 19. Compared with Figure 20, Figure 21 shows that the unbalance response of the rotor can 
be attenuated to a great extent in the given rotor operation speed range. The results mentioned further validate the effectiveness of the proposed method.

\section{Conclusions}

In the paper, unbalance compensation for an AMB system using ILC controller is introduced. The stability and steadystate tracking errors for the MIMO AMB system controlled by an ILC controller are derived. Tools used to calculate the singular values of the frequency response of a dynamic system are introduced to analyze the system stability.

Based on the concluded controller design objectives, an ILC controller is designed to attenuate the unbalance vibrations of an full scale test rig which is designed for the rotor and AMBs in HTR-10GT. With the controller, the unbalance vibrations in rotor displacements are reduced, remarkably. The control currents required in the initial iterations may lead to saturation of the power amplifier. However, this disadvantage can be avoided by adding amplitude limiting on controller outputs with low thresholds without affecting system stability. Our further research includes experimental study in real test rig and ILC controller design based on the frequency response function obtained by frequency sweeping test which will include unmodeled system dynamics.

\section{Competing Interests}

The authors declare that they have no competing interests.

\section{Acknowledgments}

This paper is financially supported by Project 61305065 which is supported by NSFC.

\section{References}

[1] H. Barnert and K. Kugeler, "HTR plus modern turbine technology for higher efficiencies," in Proceedings of a Technical Committee Meeting on Design and Development of Gas Cooled Reactors with Closed Cycle Gas Turbines, Beijing, China, 1996.

[2] Z. Wu and S. Yu, "Htgr projects in China," Nuclear Engineering and Technology, vol. 39, no. 2, pp. 103-110, 2007.

[3] G. Yang, Z. Shi, N. Mo, and L. Zhao, "Research on active magnetic bearing applied in Chinese modular high-temperature gas-cooled reactor," Progress in Nuclear Energy, vol. 77, pp. 352360, 2014.

[4] R. Herzog, P. Bühler, C. Gähler, and R. Larsonneur, "Unbalance compensation using generalized notch filters in the multivariable feedback of magnetic bearings," IEEE Transactions on Control Systems Technology, vol. 4, no. 5, pp. 580-586, 1996.

[5] K.-Y. Chen, P.-C. Tung, M.-T. Tsai, and Y.-H. Fan, "A self-tuning fuzzy PID-type controller design for unbalance compensation in an active magnetic bearing," Expert Systems with Applications, vol. 36, no. 4, pp. 8560-8570, 2009.

[6] S. Y. Yoon, L. Di, and Z. Lin, "Unbalance compensation for AMB systems with input delay: an output regulation approach," Control Engineering Practice, vol. 46, pp. 166-175, 2016.
[7] C. Hui, L. Shi, J. Wang, and S. Yu, "Adaptive unbalance vibration control of active magnetic bearing systems for the HTR-10GT," in Proceedings of the 18th International Conference on Nuclear Engineering (ICONE18 '10), 801, 793 pages, Xi'an, China, May 2010.

[8] M. Uchiyama, "Formation of high-speed motion pattern of a mechanical arm by trial," Transactions of the Society of Instrument \& Control Engineers, vol. 14, 1978 (Japanese).

[9] S. Tong, Optimal Control Using Instantaneous Optimal and Iterative Learning Control and the Convergence Analysis, Mechanics in Engineering, 2015.

[10] L. Huang, Y. Fang, and T. Wang, "Convergence analysis of wireless remote iterative learning control systems with channel noise," Asian Journal of Control, vol. 17, no. 6, pp. 2374-2381, 2015.

[11] D. Shen and Y. Wang, "Survey on stochastic iterative learning control," Journal of Process Control, vol. 24, no. 12, pp. 64-77, 2014.

[12] H.-S. Ahn, Y. Q. Chen, and K. L. Moore, "Iterative learning control: brief survey and categorization," IEEE Transactions on Systems, Man, and Cybernetics, Part C: Applications and Reviews, vol. 37, no. 6, pp. 1099-1121, 2007.

[13] Y. Wang, F. Gao, and F. J. Doyle III, "Survey on iterative learning control, repetitive control, and run-to-run control," Journal of Process Control, vol. 19, no. 10, pp. 1589-1600, 2009.

[14] H. W. Yoo, S. Ito, and G. Schitter, "High speed laser scanning microscopy by iterative learning control of a galvanometer scanner," Control Engineering Practice, vol. 50, pp. 12-21, 2016.

[15] P. C. Marchal, O. Srnmo, B. Olofsson, A. Robertsson, J. G. Ortega, and R. Johansson, "Iterative learning control for machining with industrial robots," IFAC Proceedings, vol. 47, no. 3, pp. 9327-9333, 2014.

[16] M. R. Graham, R. A. De Callafon, and L. Shrinkle, "An iterative learning controller for reduction of repeatable runout in hard disk drives," in Proceedings of the American Control Conference, Minneapolis, Minn, USA, June 2006.

[17] E. Rogers and O. R. Tutty, "Iterative learning control with applications in energy generation, lasers and health care," Proceedings of the Royal Society A: Mathematical, Physical \& Engineering Science, vol. 472, no. 2193, 2016.

[18] H. G. Chiacchiarini and P. S. Mandolesi, "Unbalance compensation for active magnetic bearings using ILC," in Proceedings of the IEEE International Conference on Control Applications (CCA '01), Cat. No.01CH37204, pp. 58-63, Mexico City, Mexico, September 2001.

[19] C. Bi, D. Wu, Q. Jiang, and Z. Liu, "Automatic learning control for unbalance compensation in active magnetic bearings," IEEE Transactions on Magnetics, vol. 41, no. 7, pp. 2270-2280, 2005.

[20] C. Bi, D. Wu, Q. Jiang, and Z. Liu, "Optimize control current in magnetic bearings using automatic learning control," in Proceedings of the IEEE International Conference on Mechatronics (ICM '04), pp. 305-310, Istanbul, Turkey, June 2004.

[21] L. Shi, S. Yu, G. Yang, Z. Shi, and Y. Xu, “Technical design and principle test of active magnetic bearings for the turbine compressor of HTR-10GT," Nuclear Engineering and Design, vol. 251, pp. 38-46, 2012.

[22] G. Schweitzer and H. Eric, Magnetic Bearings: Theory, Design, and Application to Rotating Machinery, Springer, Berlin, Germany, 2009.

[23] Cover Illustration and Mikael Norrlof, Iterative learning control—analysis, design, and experiments, 2000. 
[24] G. Wang and Z. He, Applied $H_{\infty}$ Control, Harbin Institute of Technology Press, 2010.

[25] C. R. Phillips and N. T. Nagle, Digital Control System Analysis and Design, Prentice Hall, Upper Saddle River, NJ, USA, 1990. 

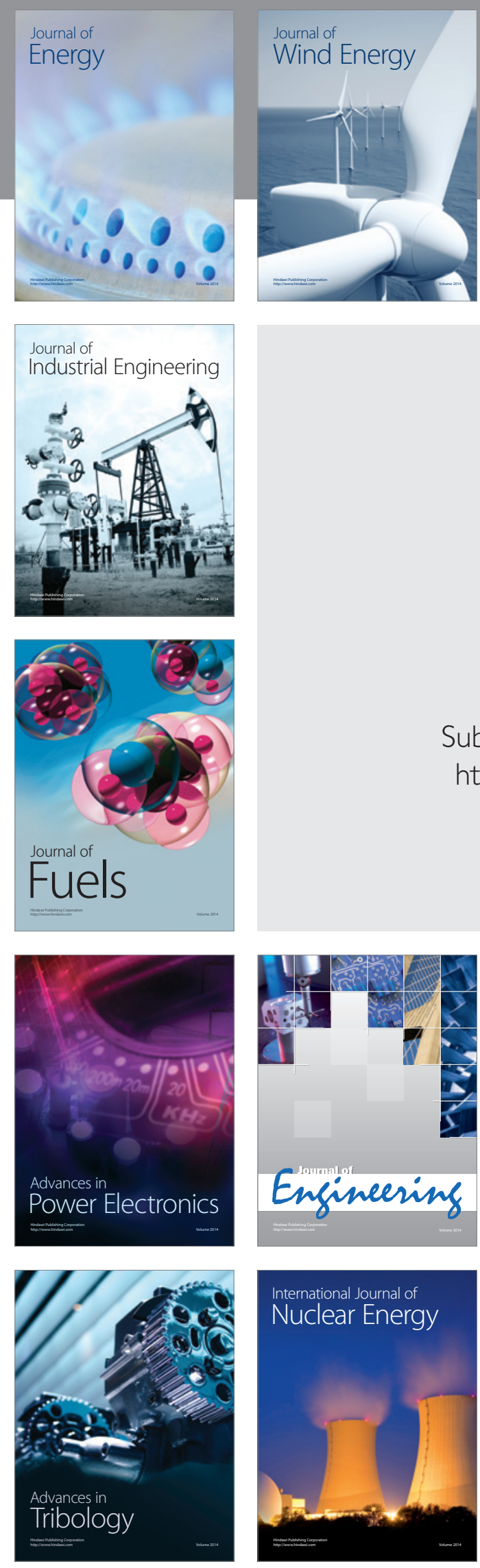

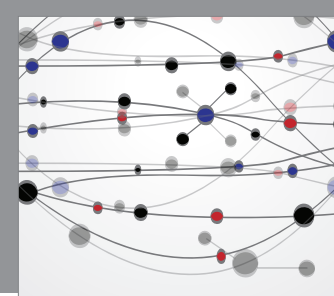

The Scientific World Journal
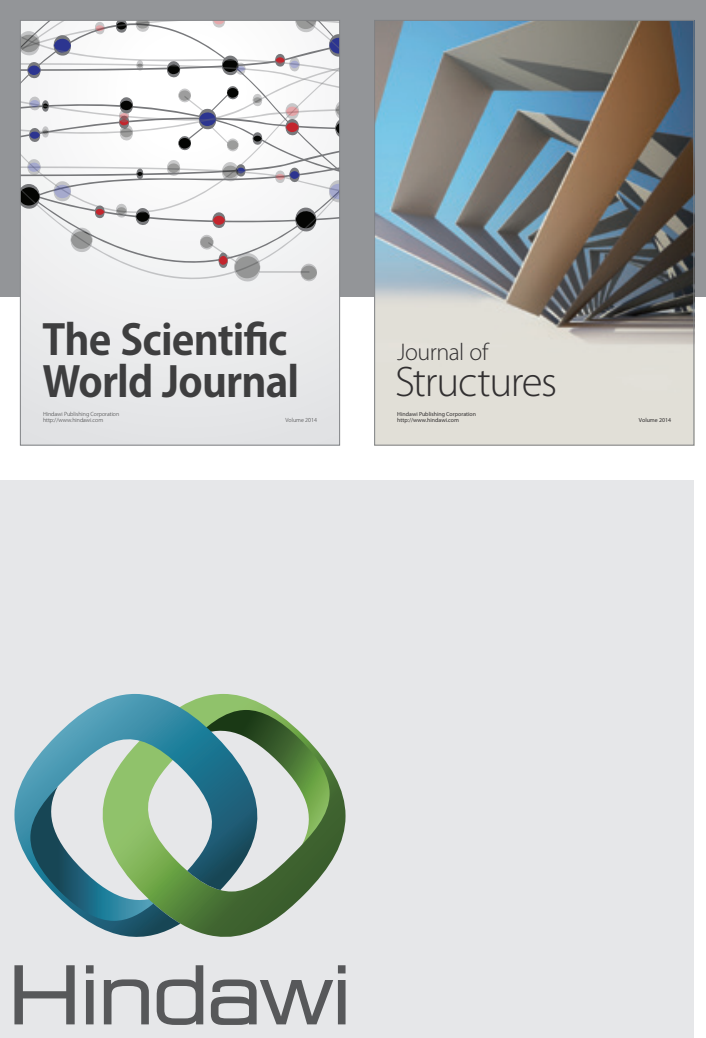

Submit your manuscripts at

https://www.hindawi.com
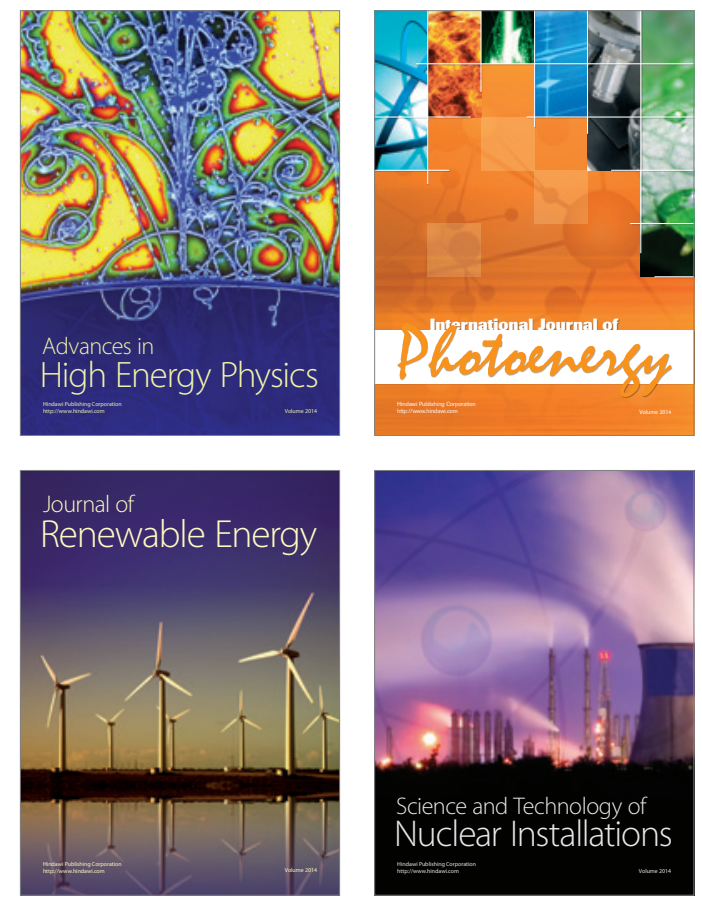
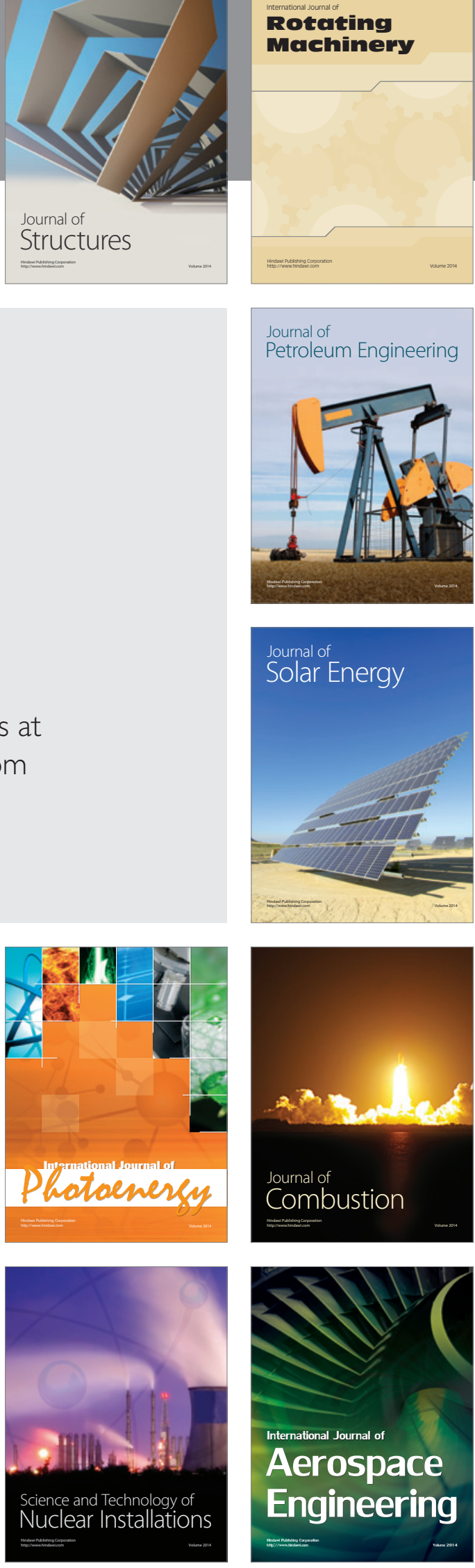